\title{
Motor Learning Drives Dynamic Patterns of Intermittent Myelination on Behaviorally-Activated Axons
}

\author{
Clara M. Bacmeister ${ }^{1}$, Rongchen Huang ${ }^{2,3}$, Michael A. Thornton ${ }^{1}$, Lauren Conant ${ }^{1}$, Anthony Chavez ${ }^{1}$, \\ Alon Poleg-Polsky ${ }^{3}$, Ethan G. Hughes ${ }^{1,4}$
}

\begin{abstract}
Myelin plasticity occurs when newly-formed and pre-existing oligodendrocytes remodel existing myelination. Recent studies show these processes occur in response to changes in neuronal activity and are required for learning and memory. However, the link between behaviorally-relevant neuronal activity and circuit-specific changes in myelination remains unknown. Using longitudinal, in vivo two-photon imaging and targeted labeling of behaviorally-activated neurons, we explore how the pattern of intermittent myelination is altered on individual cortical axons during learning of a dexterous reach task. We show that learning-induced plasticity is targeted to behaviorally-activated axons and occurs in a staged response across cortical layers. During learning, myelin sheaths retract, lengthening nodes of Ranvier. Following learning, addition of new sheaths increases the number of continuous stretches of myelination. Computational modeling suggests these changes initially slow and subsequently increase conduction speed. Thus, behaviorallyactivated, circuit-specific changes to myelination may fundamentally alter how information is transferred in neural circuits during learning.
\end{abstract}

In the CNS, oligodendrocytes extend numerous processes and enwrap nearby axons with discrete myelin sheaths. Myelin is traditionally known for its role in saltatory conduction, where its insulating properties accelerate action potential propagation by allowing local electrical circuits only to be generated at nodes of Ranvier ${ }^{1}$. However, the discovery of intermittently myelinated axons ${ }^{2}$, with large gaps between individual myelin sheaths, raises the possibility that sheath location and diverse patterns of myelination could represent an additional avenue for which experience-dependent plasticity could modulate signal transmission between neurons.

While myelination begins during prenatal development, this process continues throughout life and is correlated with the functional maturation of specific brain circuits ${ }^{3}$. Recent work shows that neuronal activity and naturalistic experiences during adulthood regulate the formation of new myelin by modulating the generation of adult-born oligodendrocytes ${ }^{4,5}$. Importantly, this process is required for learning novel behaviors and the consolidation of new memories $^{6-9}$. However, the link between behaviorally-relevant neuronal activity and circuit-specific changes in myelination remains unknown.

Recent studies show that neuronal activity can regulate myelin sheath thickness, length, and placement during oligodendrocyte generation and maturation. Exogenous stimulation or interruption of neuronal activity biases the selection of axons targeted for myelination ${ }^{10-12}$. Furthermore, neuronal activity regulates elongation by modulating calcium signaling within nascent myelin sheaths ${ }^{13,14}$. These types of adjustments during myelin sheath generation and placement can be used to fine tune communication between neurons by modifying timing delays ${ }^{15-17}$ and synchronizing signals from distant areas ${ }^{18}$ during the functional maturation of these circuits.

Long thought to be a stable structure once generated, studies show that extrinsic signals can remodel pre-existing myelin sheaths and nodes of Ranvier outside of the initial myelin generation process. Following oligodendrocyte differentiation, the length of myelin sheaths is largely established in 3-7 days ${ }^{19,20}$. However, in developing zebrafish, mature myelin sheaths continue to elongate to compensate for body growth and ablation of neighboring sheaths induces remodeling of remaining sheaths ${ }^{19}$. Recent studies suggest that exogenous stimulation or spatial learning can modify the length of node of Ranvier, altering conduction velocity ${ }^{21}$. Moreover, sensory deprivation induces experience-dependent remodeling of pre-existing sheaths on distinct neuronal subclasses ${ }^{22}$.

However, our understanding of the consequences and implications of modifying myelination as a form of neural plasticity remains incomplete. Whether myelin is modified in specific neuronal circuits during learning or life experience is entirely unknown. Furthermore, how changes in new and pre-existing myelination translate into computational alterations required for modulating behavioral output remains poorly understood. The intermittent pattern of myelination, which persists throughout life ${ }^{5}$, may be an anatomical anomaly in the adult CNS, or alternatively, it may represent a distinct and dynamic design used to fine tune the function of neuronal circuits underlying learning and behavior.

Through longitudinal, in vivo imaging of individual oligodendrocytes, myelin sheaths, and neuronal axons in behaviorally-activated neuronal ensembles, we show that myelin and nodal plasticity are specifically targeted to axons involved in learning a novel forelimb reach task. Computational modeling predicts that retraction of consecutive pre-existing myelin sheaths and lengthening of pre-existing nodes during learning slows conduction, while the addition of new sheaths speeds conduction in the weeks after learning. Together, these results suggest that phasic shifts in the pattern of axonal myelination within behaviorallyrelevant circuits alter neuronal computations underlying modified behavioral outputs during learning.

\section{Results}

Learning modulates nodes of Ranvier in a staged response.

Individual cortical axons are intermittently myelinated, where large stretches of exposed axon are interspersed with regions of myelination $^{2}$. While previous work clearly demonstrates that oligodendrogenesis is altered by learning ${ }^{6,20}$, how these changes affect the pattern of myelin and nodes of Ranvier on individual axons remains unclear. To determine changes to the pattern of nodes of Ranvier on individual axons over time, we used longitudinal in vivo two-photon imaging in the forelimb region of motor cortex of $M O B P-E G F P$ transgenic mice, which express EGFP in all mature oligodendrocytes and their associated myelin sheaths in corte $\mathrm{x}^{5,20}$. We trained 2-3 month old $M O B P-E G F P$ mice in a skilled, single pellet contralateral forelimb reach task (Supplementary Fig. 2) and conducted long-term in vivo imaging and post-hoc immunostaining. With near-infrared branding ${ }^{23}$, we aligned longitudinal, in vivo images of myelin and nodes with post- hoc immunostained axons to 
a

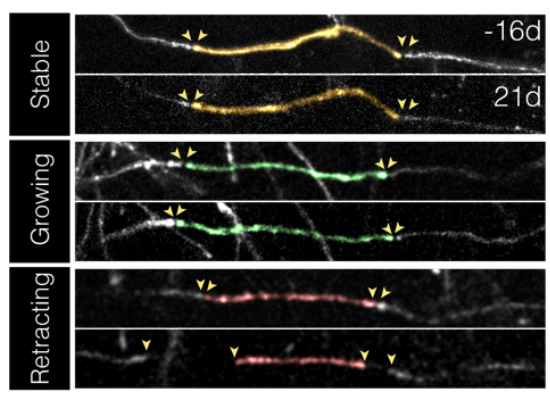

b

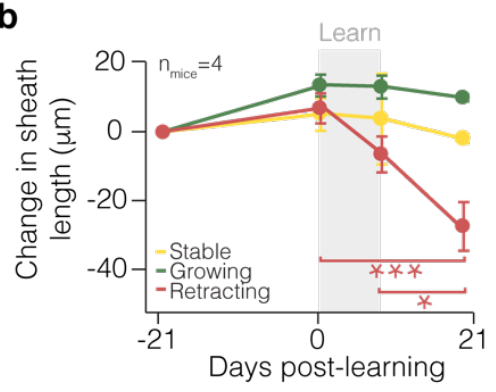

1 neighbor

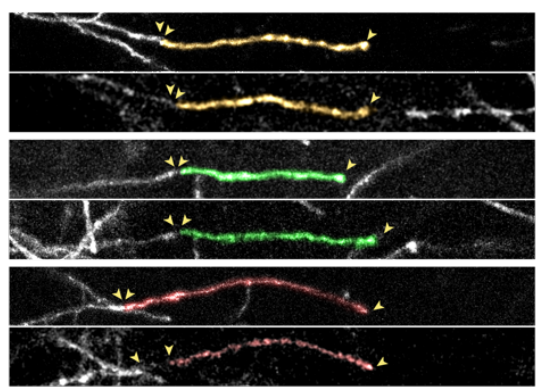

C

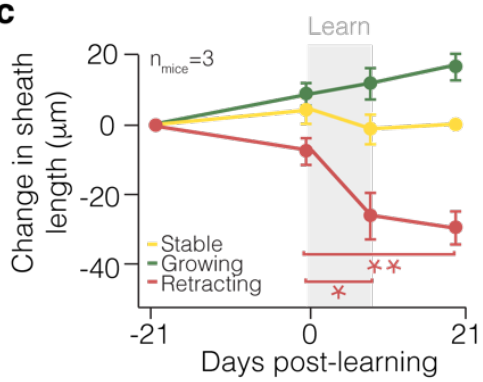

0 neighbors

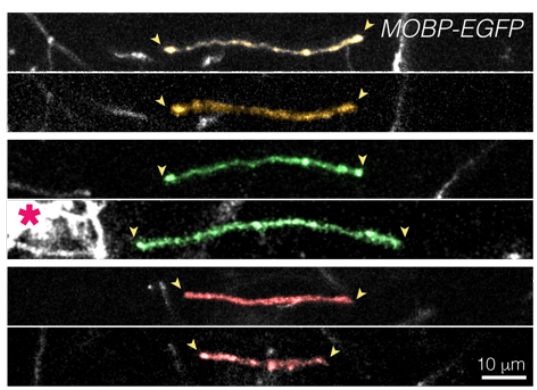

d

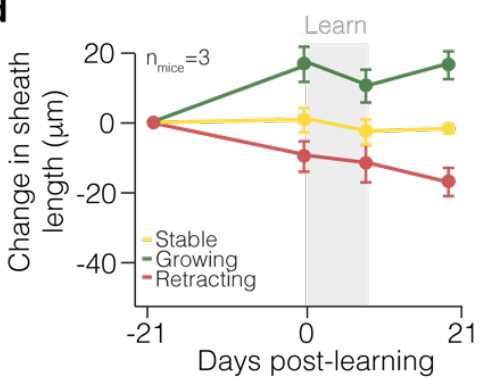

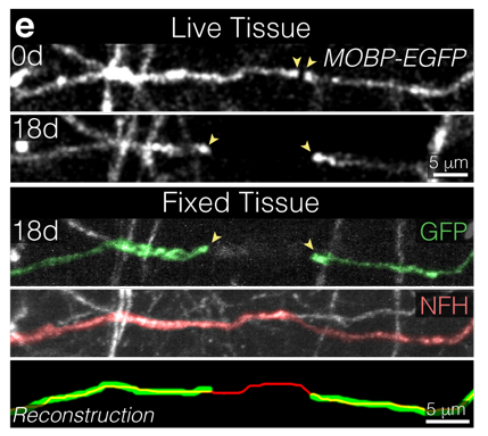

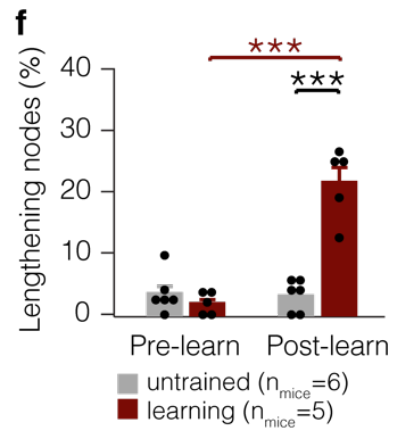

g

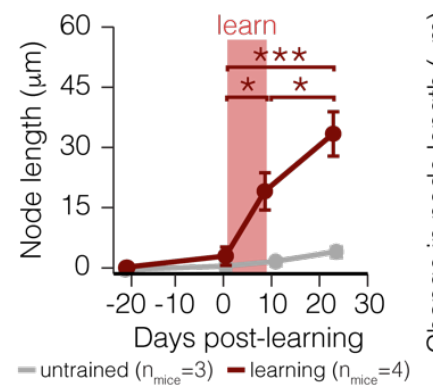

h

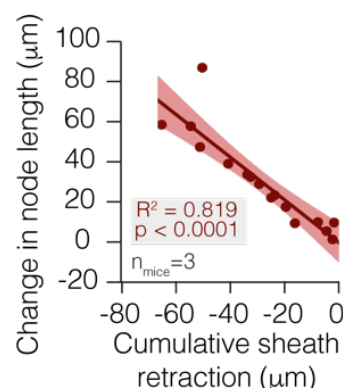

Figure 1 | Learning-induced sheath retraction modifies node length. a, All types of sheaths exhibit three behaviors: stable (top), growing (middle), and retracting (bottom). Note newly-generated oligodendrocyte (middle-right pink asterisk). b, Retraction of sheaths with two neighbors is specifically modulated by learning $\left(F_{3,48}=9.04, p<0.0001\right)$ and continues for two weeks after learning (0d vs. $21 d, p=0.0001 ; 9 d$ vs. $21 d, p=$ 0.021 ; Tukey's HSD). c, Retraction of sheaths with one neighbor is specifically modulated by learning $\left(F_{3,40}=9.93, p<0.0001\right)$ and finishes within the learning period ( $p=0.029$; Tukey's HSD). $\mathbf{d}$, Dynamics of sheaths with 0 neighbors are not specifically modulated by learning. e, A longitudinally-imaged, lengthened node before (0d) and after learning (18d), and that same node in fixed and sectioned tissue stained for GFP (green) and neurofilament (NFH, red). The bottom panel shows a reconstruction of the lengthened node and axon. f, Learning modulates proportion of nodes lengthening $\left(F_{1,14}=438.85, p=0.0002\right)$. More nodes lengthen after learning than before learning in mice that receive motor learning ( $p<0.0001$; Tukey's HSD). More nodes lengthen in learning mice than in untrained mice $(p<0.0001$; Tukey's HSD). g, Learning modulates node length $\left(\mathrm{F}_{3,83.38}=4.88, p=0.0036\right)$. Nodes increase significantly in length during learning $(p=0.039$; Tukey's HSD) and continue to lengthen following learning $(p=0.03$; Tukey's HSD). $\mathbf{h}$, Changes in node length are significantly correlated to changes in sheath length of associated myelin sheaths $(p<0.0001) .{ }^{*} p<0.05$, ${ }^{* *} p<0.01,{ }^{* * *} p<0.0001$, NS, not significant; bars and error bars represent mean \pm s.e.m. For detailed statistics, see Supplementary Table 3, Figure 1.

resolve dynamics of pre-existing and newly generated nodes and myelin sheaths along individual axons in response to learning (Supplementary Fig. 1). Age-matched, untrained controls were exposed to the training environment without completing the behavioral task and received the same diet as learning mice for the duration of the experiment.

In the cortex, intermittently myelinated axons possess three types of sheaths that are defined by their location relative to neighboring myelin: sheaths with two directly neighboring myelin sheaths, sheaths with one directly neighboring myelin sheath, and sheaths with no direct neighbors (Fig. 1a). In all cases, "direct neighbors" are defined as sheaths that form nodes of 3 microns or less with each other. Pre-existing sheaths showed three different behaviors, either maintaining their length for the duration of the study (stable), lengthening over time (growing), or shrinking over time (retracting). While we found growing sheaths across all timepoints, only sheath retraction was altered in learning mice relative to untrained mice (Supplementary Fig. 3). Next, we examined the dynamics of the three types of sheaths on intermittently myelinated axons (Fig. 1a). When we examined specific sheath behaviors across time, we found that retraction of pre-existing myelin sheaths was specifically regulated by learning in sheaths with either one or two neighbors (Fig. 1b,c). Retraction of sheaths with two neighbors was protracted and continued for two weeks after learning (Fig. 1b), in contrast to the retraction of sheaths with one neighbor, which occurred within the week of task acquisition (Fig. 1c). The growth and retraction of sheaths without neighbors was not regulated by learning (Fig. 1d). Therefore, learning specifically modulates the retraction of continuous stretches of myelin - that is, areas in which myelin sheaths form nodes with one or two directly neighboring sheaths. In contrast, in regions of where sheaths do not have any direct neighbors and therefore do not have any associated nodes, sheath retraction is not specifically modulated by learning. 

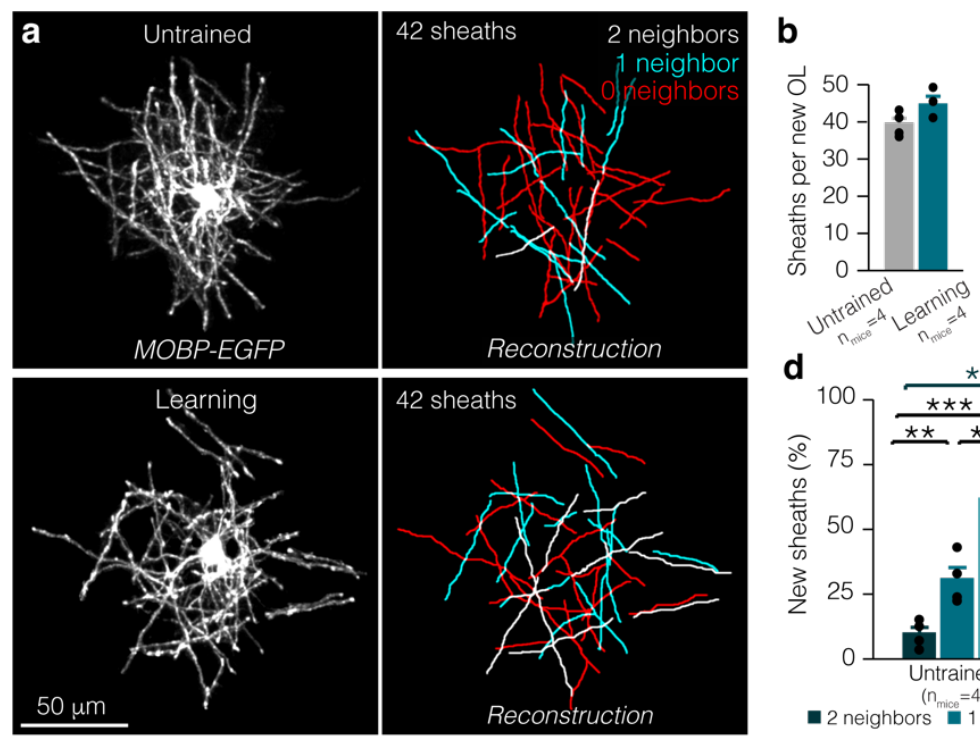

C
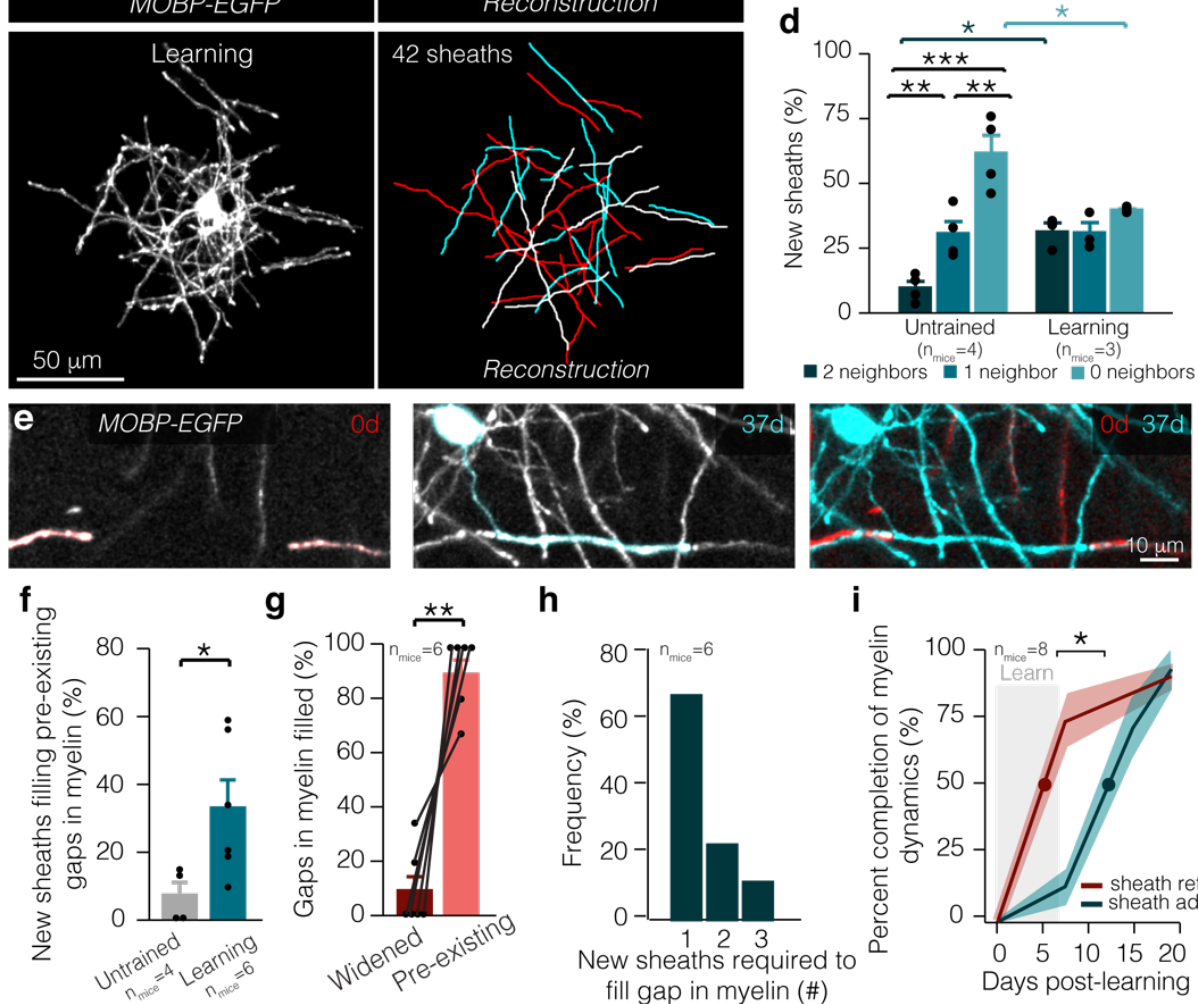

g
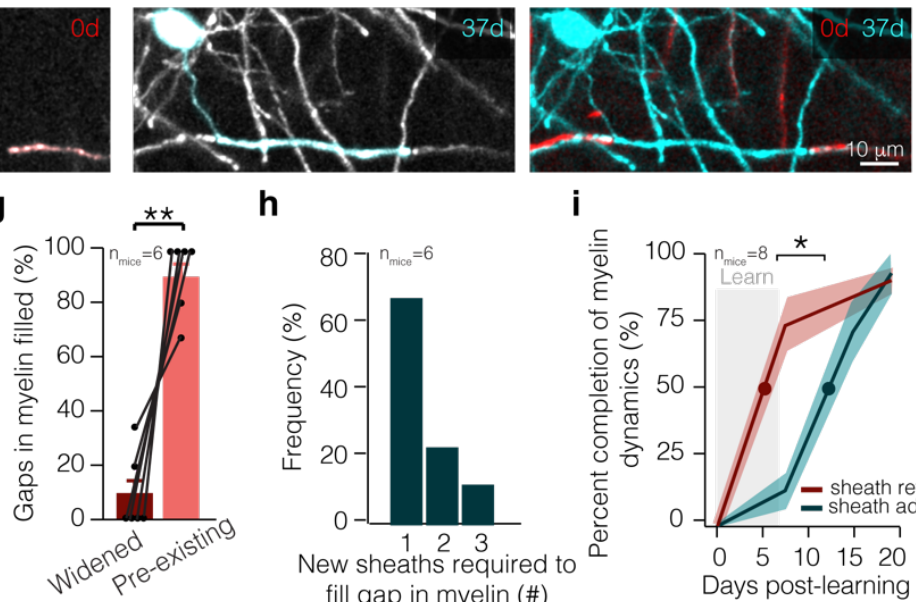

h

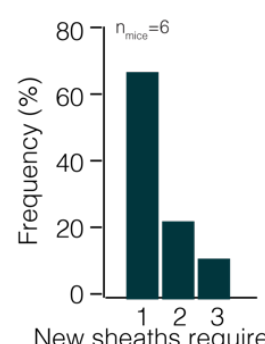

New sheaths required to

fill gap in myelin (\#) i

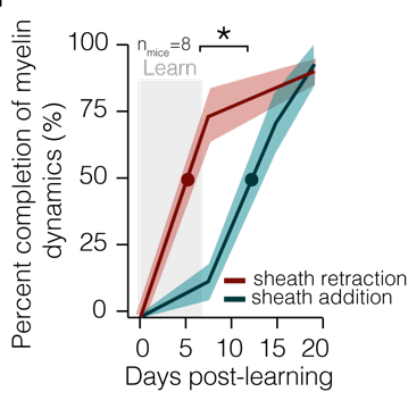

Figure 2 | Myelin and nodal plasticity occur in distinct phases in response to learning. a, An entire arbor of an individual oligodendrocyte in vivo (left) and in reconstruction (right), in untrained (top) and learning (bottom) MOBPEGFP mice. b, No difference in sheath number per new oligodendrocyte in untrained and learning mice. c, No difference in sheath length on new oligodendrocytes in untrained and learning mice. Bars and error bars represent median and I.Q.R. d, Learning modulates the types of sheaths produced by new oligodendrocytes $\left(F_{5,15}=15.03, \quad p<0.0001\right)$. Continuous sheath production is heightened after learning ( $p=0.048$; Tukey's HSD), while new oligodendrocytes produce less isolated sheaths $(p=0.044$; Tukey's HSD). e, A new sheath (blue, 37d) fills in a gap in pre-existing myelination (red, 0d) after learning (37d). f, After learning, more sheaths per new oligodendrocyte fill in pre-existing gaps in myelination (Student's t-test, $\mathrm{t}(6.85)=-2.78$, $\mathrm{p}=0.028$ ). $g$, Following learning, the majority of newly filled gaps in myelination are pre-existing gaps in myelination rather than nodes that lengthened (Paired Student's t-test, $t(5)=6.99, p=0.0009)$. $h$, The majority pre-existing gaps in myelination are filled in by one sheath, but up to three new sheaths can be responsible for filling in a gap in pre-existing myelination. i, Learning-induced sheath retraction precedes the post-learning burst in oligodendrogenesis and new sheath addition (Student's $t$-test, $t(13.43)=-2.46$, $\mathrm{p}=0.028) . \quad{ }^{*} \mathrm{p}<0.05, \quad{ }^{* *} \mathrm{p}<0.01$ ${ }^{* * *} p<0.0001$, NS, not significant; bars and error bars represent meants.e.m. For detailed statistics, see Supplementary Table 3, Figure 2.
Since pre-existing, continuous myelin sheaths respond to learning by retracting, we reasoned that the length of their associated nodes may also be modulated. To explore this possibility, we conducted post-hoc immunohistochemistry on myelin sheaths, nodes, and their associated axons in mice that received longitudinal in vivo two-photon imaging (Fig. 1e; Supplementary Fig. 1,4). When we examined node dynamics in response to learning, we found that $21.73 \pm 1.61 \%$ of pre-existing nodes of Ranvier elongated after learning, whereas we did not find heightened node lengthening in untrained mice (Fig. 1f). These learning-induced sheath dynamics resulted in an average increase in node length to $18.63 \pm 4.62$ microns during reach task acquisition. In mice that engaged in motor learning, nodes continued to lengthen in the two weeks following learning, causing an additional increase in length of $14.31 \pm 5.50$ microns (Fig. 1g). Changes to node length were highly correlated to the changes in myelin sheath length (Fig. 1h), indicating that sheath retraction plays an integral role in node length modification.

Learning exposed regions of previously myelinated axon via sheath retraction, however, pre-existing sheaths did not grow to add myelin to regions of exposed axon. To explore how learning might increase myelination of cortical axons, we examined how sheath addition by newly generated oligodendrocytes changed the myelination pattern in the cortex in the weeks following learning. Learning did not affect the number of sheaths per individual oligodendrocyte nor the length of these sheaths (Fig. 2a-c). However, we found that learning influenced where newly generated oligodendrocytes placed their myelin. In untrained mice, new oligodendrocytes generated primarily isolated sheaths, however, after learning, new oligodendrocytes generated an increased number of continuous and fewer isolated myelin sheaths (Fig. 2a,d). Sheath addition after learning was more likely to be added to partially myelinated regions of an axon, filling in gaps in pre-existing myelin to create continuous stretches of myelination (Fig. 2e,f). These newly-filled gaps in myelination were not nodes that lengthened in response to learning. Instead, new sheaths filled in large gaps in the pattern of pre-existing myelin that were present even before learning occurred (Fig. 2g). In 6/18 of these cases, multiple new sheaths were placed consecutively to fill in a gap in pre-existing myelination pattern (Fig. 2h). The addition of new myelin sheaths in response to learning was delayed by $5.93 \pm 2.41$ days relative to learning-induced sheath retraction, which began during learning (Fig. 2i). Together, these results indicate that motor learning-induced myelin plasticity proceeds in a phasic response in forelimb motor cortex, first by causing retraction of pre-existing myelin sheaths during learning followed by the addition of new sheaths to form continuous stretches of myelination after learning.

\section{A subpopulation of axons exhibits nodal dynamics in response to learning.}

We next sought to determine whether learning-induced changes to myelin and nodal architecture were driven by signals intrinsic or extrinsic to oligodendrocytes (Fig. 3). We found that remodeling of myelin sheaths was not specific to individual oligodendrocytes; in fact, the majority of oligodendrocytes in both the untrained and 
a
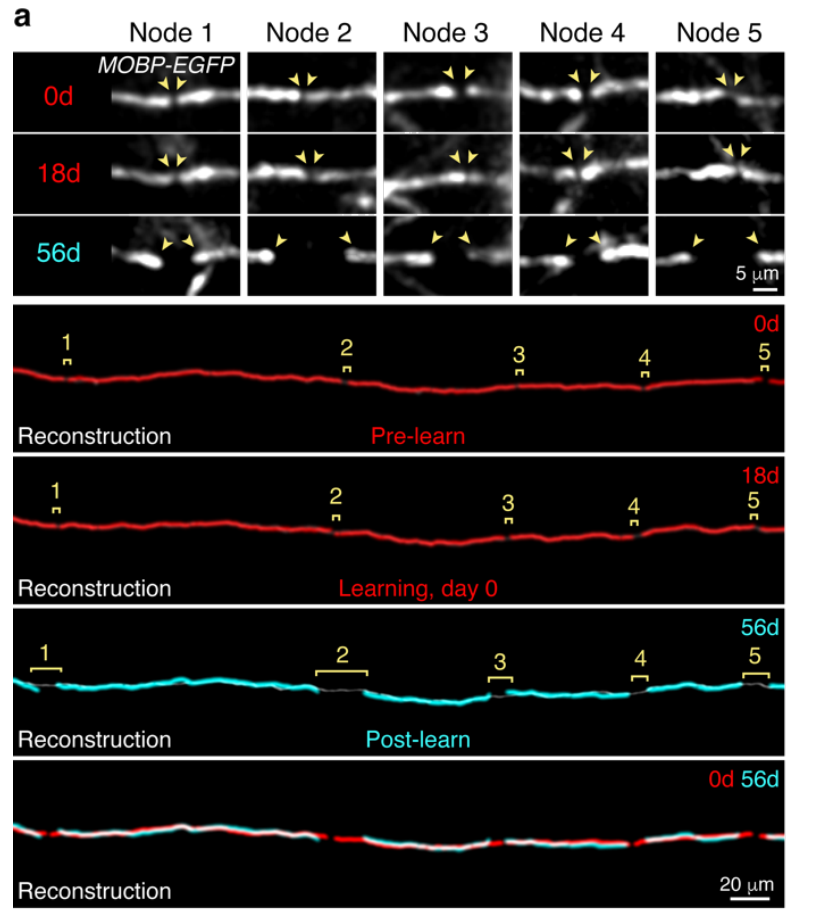

d

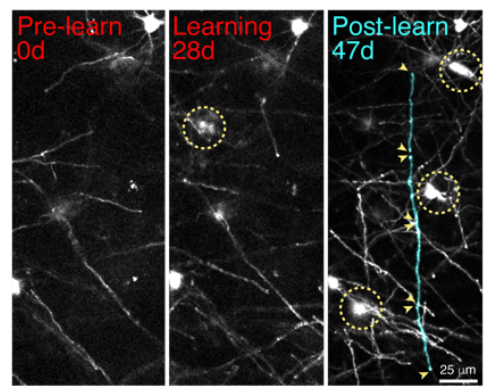

e

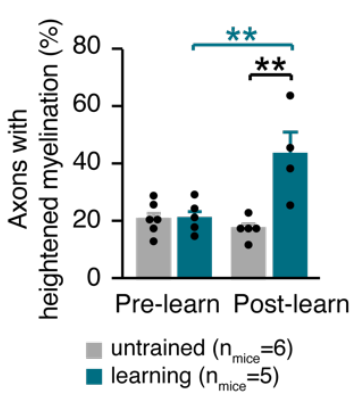

b

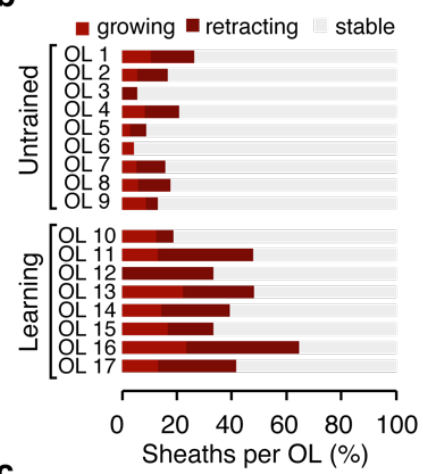

c

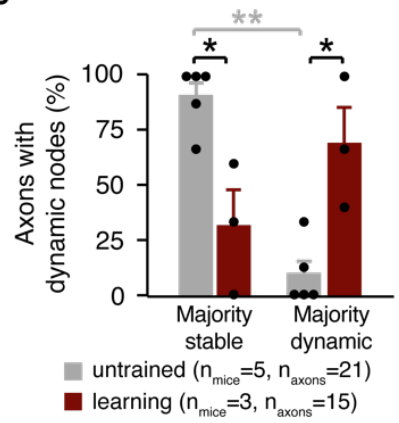

f

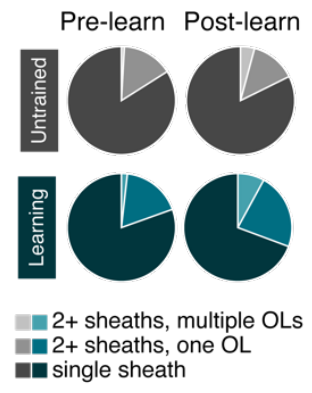

Figure $3 \mid$ Learning-induced changes to myelin are axonspecific. a, Multiple nodes along the same axon lengthen in response to learning (in vivo data shown on top, reconstructions shown on bottom). b, Oligodendrocytes in learning and untrained mice exhibit multiple sheath behaviors. c, Learning modulates the proportion of axons with multiple dynamic nodes $\left(\mathrm{F}_{3,12}=14.17, \mathrm{p}=\right.$ 0.0003 ). In learning mice, significantly more axons with dynamic myelin possess multiple dynamic nodes than in untrained mice $(p=0.01$; Tukey's HSD). d, Axons can be myelinated by multiple sheaths. e, Learning modulates the proportion of axons with heightened myelination $\left(F_{1,9.46}=14.17\right.$, $p=0.0027)$. After learning, axons are more often myelinated by multiple new sheaths than before learning $(\mathrm{p}=$ 0.0055 ; Tukey's HSD). The percent of axons with heightened myelination is increased in learning compared to untrained mice ( $p=0.006$; Tukey's HSD). f, Proportion of axons receiving new myelin from multiple oligodendrocytes, multiple sheaths from a single oligodendrocyte, or only one new sheath in untrained mice (gray) and before and after learning (blue). ${ }^{*} p<0.05,{ }^{* *} p<0.01,{ }^{* * *} p<$ 0.0001 , NS, not significant; bars and error bars represent mean \pm s.e.m. For detailed statistics, see Supplementary Table 3, Figure 3.

learning conditions possessed stable, retracting, and growing sheaths (14/17 oligodendrocytes; Fig. 3b). In contrast, learninginduced changes in nodal length were concentrated on individual axons with changes to node length occurring at multiple nodes along individual axons (Fig. 3a,c). In untrained mice, dynamic nodes were distributed across the axonal population and the majority of nodes along individual axons remained stable. In contrast in learning mice, individual axons had dynamic nodes that were concentrated along their length constituting the majority of their nodes (Fig. 3c). These findings indicate that nodes are modified in concert across select stretches of continuous myelin on individual axons in response to learning.

To determine whether sheath addition was similarly targeted to specific axons after learning, we examined the placement of myelin sheaths from newly generated oligodendrocytes. Before learning, new oligodendrocytes myelinated similar numbers of axons as in untrained mice, however, after learning, new oligodendrocytes placed more myelin on the same axons in trained mice compared to untrained mice (Fig. 3d,e). These axons could receive multiple, consecutive sheaths from both individual oligodendrocytes and from more than one oligodendrocyte (Fig. 3f). Overall, we find that targeted sheath retraction generates new patterns of intermittent myelination during learning. After learning, we find the addition of new sheaths to unmyelinated gaps heightens the formation of continuous stretches of myelination. Together, these results indicate that motor learning specifically modulates

patterns of intermittent myelination on select subsets of axons in the primary motor cortex.

Learning induces myelin and nodal dynamics broadly across cortical layers.

Previously, we showed that motor learning induced layer-specific changes in oligodendrogenesis in the primary motor cortex ${ }^{20}$. Our current data replicated this finding as oligodendrogenesis increases in Layer 1 (L1) but not layer 2/3 (L2/3) following the forelimb reach task $(0.72 \pm 0.02$ vs. $1.35 \pm 0.09 \%$ in L1 of untrained vs. learning mice; $\mathrm{p}=0.016$ ). To examine whether these layer-specific differences are paired with axon-specific effects on myelin and nodal plasticity following learning, we analyzed pre-existing nodes and new sheath dynamics in layers 1-3 in the primary forelimb motor cortex. In L1, on mostly continuously myelinated axons, we found an increased proportion of lengthening nodes after learning, relative to node dynamics before learning and relative to nodes in untrained mice (Fig. 4a,b). Node lengthening occurred in concert along the length of individual axons in mice that learned the forelimb reach task compared to untrained mice (Fig. 4c). Similarly, the proportion of individual axons myelinated by multiple new sheaths was also increased following learning in L1 (Fig. 4a,d). In L1, the addition of new myelin in response to learning was delayed by $\sim 4$ days $(4.27 \pm 1.08$ days) relative to learning-induced node lengthening, which began during learning (Fig. 4e). These results indicate that the learning-induced myelin and node changes we observed broadly across the superficial cortex are indeed present in 
bioRxiv preprint doi: https://doi.org/10.1101/2021.10.13.464319; this version posted October 15, 2021. The copyright holder for this preprint (which was not certified by peer review) is the author/funder. All rights reserved. No reuse allowed without permission.

a
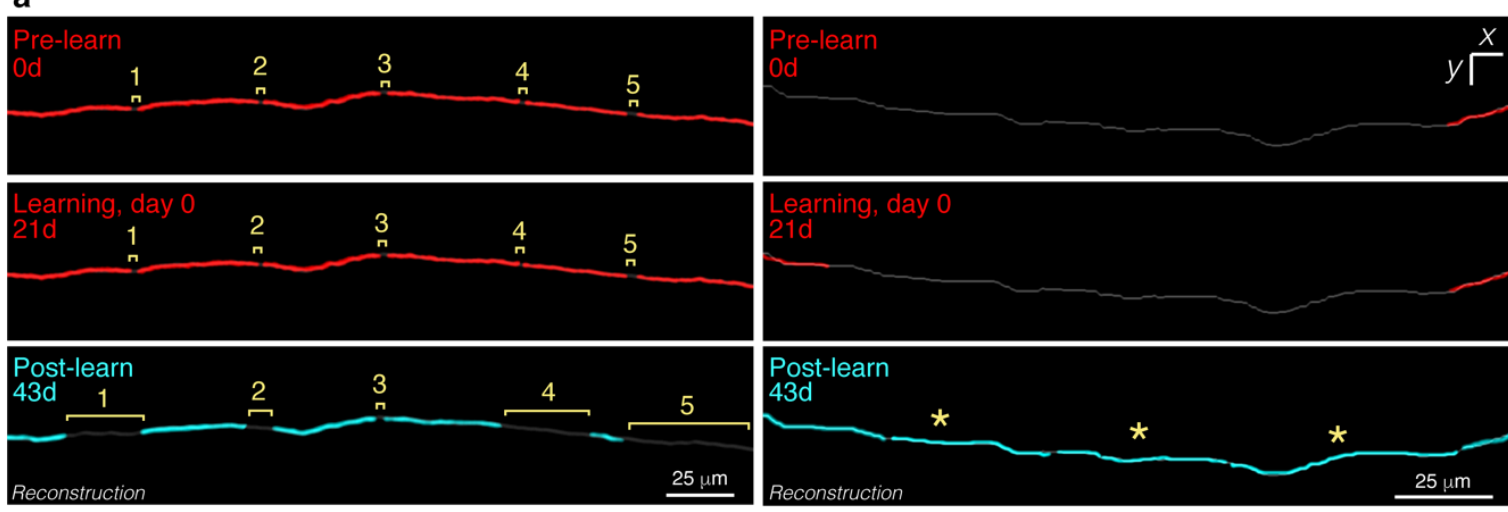

b
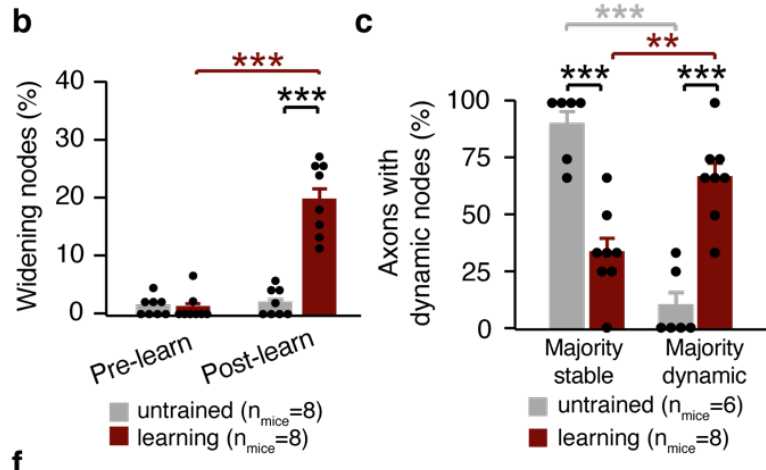

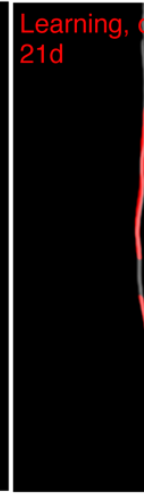

h

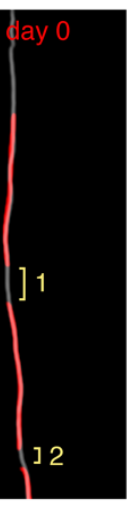

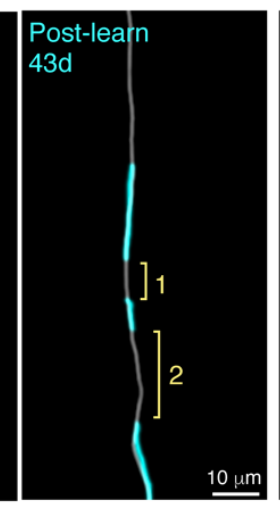

.
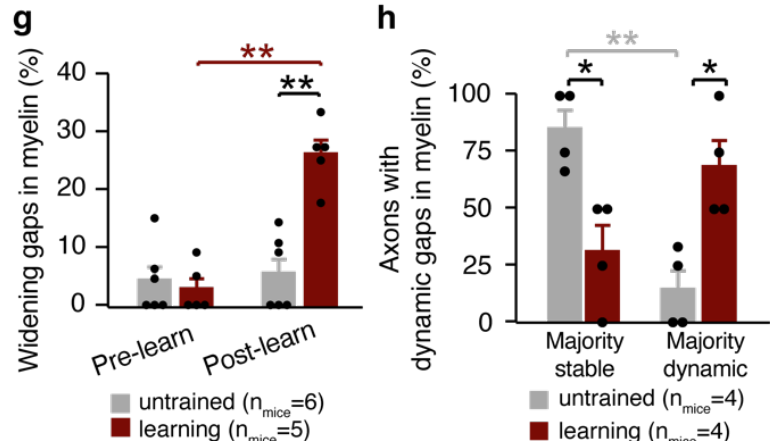

d

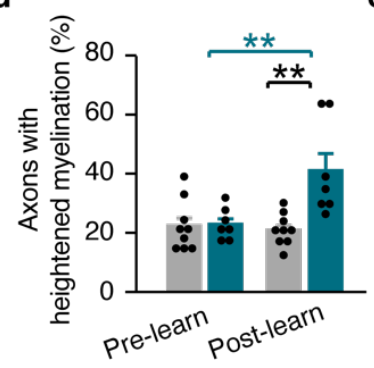

untrained $\left(n_{\text {mice }}=9\right)$

learning $\left(\mathrm{n}_{\text {mice }}=7\right)$

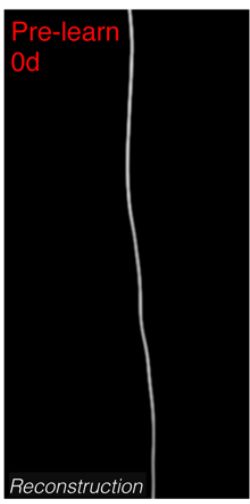

i

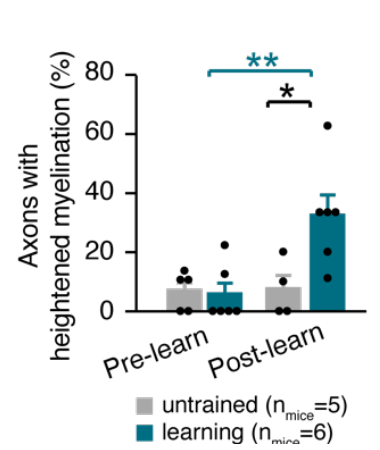

e

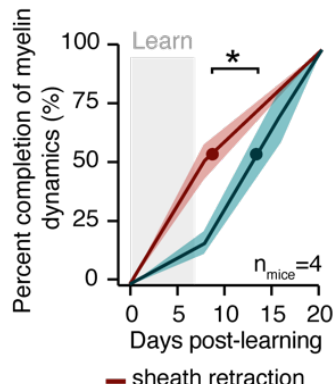

= sheath retraction
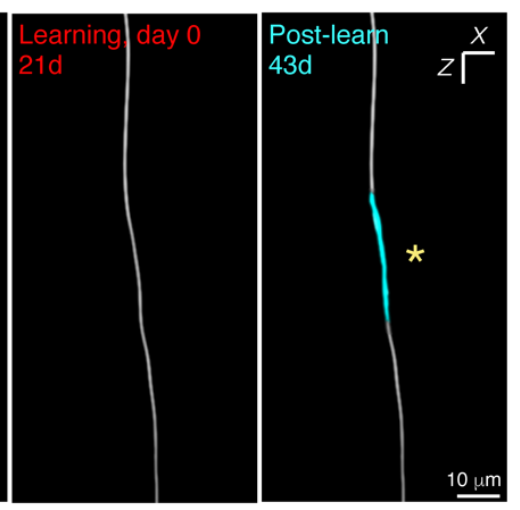

j

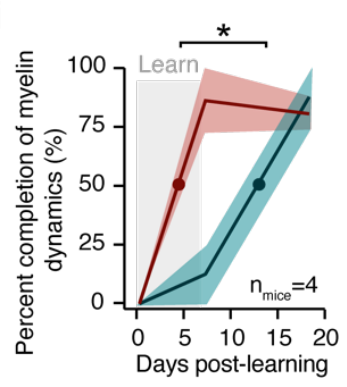

= sheath retraction

Figure 4 | Learning induces myelin and nodal dynamics broadly across cortical layers. a, Reconstructions of axons with multiple nodes lengthening (left) and heightened sheath addition (right) following learning in L1. b, Learning modulates node lengthening in $L 1\left(F_{1,1}=49.33, p<0.0001\right)$. More nodes lengthen after than before learning in L1 ( $<<0.0001$; Tukey's HSD) and compared to untrained mice in L1 ( $p<0.0001$; Tukey's HSD. and L2/3 $(p=0.0014$; Tukey's HSD). c, Learning modulates the proportion of axons with multiple dynamic nodes in $L 1\left(F_{3,24}=25.12, p<0.0001\right)$. In learning mice, significantly more axons with dynamic myelin possess multiple dynamic nodes than in untrained mice in L1 ( $p<0.0001$; Tukey's HSD). In L1, learning mice have significantly more axons with multiple dynamic nodes than a single dynamic node ( $p=0.0052$; Tukey's HSD). Legend continued on next page.

L1.

Due to the sparse myelination of L2/3 axons ${ }^{2}$ nodes of Ranvier between continuous myelin sheaths were relatively rare in
L2/3 compared to L1 (39.5 vs. $76.5 \%$ of sheaths forming nodes respectively). Therefore, to analyze sheath retraction, we analyzed whether the distance between myelin sheaths (rather than nodes) 
was altered by motor learning. Like in L1, pre-existing gaps in myelin in L2/3 lengthened in response to learning, exposing large areas of the previously myelinated axonal membrane (Fig. $\mathbf{4 f , g}$ ). Similar to changes in the more heavily myelinated L1 axons, in L2/3 unmyelinated gaps along the length of the same axon lengthened in concert in learning mice, whereas axons in untrained mice had largely stable myelin profiles (Fig. $\mathbf{4 h}$ ). Furthermore, while the rate of oligodendrogenesis was unaltered by learning in L2/3, we found that the proportion of axons receiving new myelin sheaths was increased following learning suggesting targeted placement of newly generated myelin sheaths (Fig. 4f,i). In L2/3, the addition of new myelin in response to learning was delayed by $\sim 7.5$ days $(7.58$ \pm 2.61 days) relative to sheath retraction, the majority of which was completed during learning (Fig. 4j). The delay between sheath retraction and sheath addition in L2/3 was not statistically different from the delay in L1 $(p=0.058)$. Together, these results show that similar dynamics of targeted sheath retraction, node lengthening, and sheath addition occur across layers 1-3 of superficial cortex in response to learning in spite of varying levels of myelination and axons from distinct neuronal class subtypes. Overall, these findings suggest that motor learning can drive changes in myelination irrespective of changes in rates of oligodendrogenesis.

\section{Predicted effect of learning-induced nodal dynamics on action potential propagation.}

Recent evidence indicates that changes to nodal and myelin patterning can alter conduction velocity ${ }^{16,17,21,24}$. To explore the functional implications of axon-specific myelin and nodal plasticity during motor learning, we used the NEURON platform to simulate action potential propagation in a multicompartmental model of a myelinated axon. Previous work shows that spike propagation in demyelinated regions depends on axonal excitability, specifically the imbalance of sodium:leak conductance ${ }^{25}$. To test the effect of learning-induced node lengthening on axonal function, we modulated sodium and leak conductance (gNa and gL, respectively) along newly-exposed unmyelinated regions at three lengths: 1, 20, 35 , and 184 microns (the average length of dynamic nodes prelearning, directly following learning, two weeks post-learning, and the largest empirically observed exposed axon filled by new myelin, respectively (see Fig.1, Supplemental Fig. 5, see Methods, Table 1 for a detailed summary of model and parameters).

In the pre-learning conditions, action potential conduction speed was greater than $2 \mathrm{~m} / \mathrm{s}$. As nodes lengthened, conduction speeds slowed, and failures became more prominent (Fig. 5a-c). We categorized the propagation across the unmyelinated gap in one of three ways: conduction speed above $2 \mathrm{~m} / \mathrm{s}$ ("Success"), slower conduction speed ("Delayed"), and failure to propagate ("Failure") (Fig. 5a). Unexpectedly, modification of node lengths to 20 microns resulted in propagation delays in more than $75 \%$ of the examined $\mathrm{gNa}: \mathrm{gL}$ ratios (Fig. 5c,d). Correspondingly, almost all spikes were either delayed or failed to propagate at larger node lengths (Fig. $\mathbf{5 c , d}$ ). These results imply that sheath retraction, node lengthening, and modulation of ion channel distributions along newly lengthened nodes is an effective way to alter conduction velocity.
Experimental observations revealed that up to 7 adjacent nodes could be modified in mice engaged in motor learning (see Fig. 3c, Supplemental Fig. 5). To test the effect of such numerous nodal dynamics on conduction speed, we simulated the lengthening of adjacent nodes by retracting their associated sheaths (Fig. 5e; Supplemental Fig. 5). We focused on 4 separate gNa : gL conductance ratios $\left(0.4 \mathrm{~S} / \mathrm{cm}^{2}\right.$ or $3.4 \mathrm{~S} / \mathrm{cm}^{2}$ for sodium conductance; $0.01 \mathrm{~S} / \mathrm{cm}^{2}$ or $0.08 \mathrm{~S} / \mathrm{cm}^{2}$ for leak conductance; Fig. 5e). When seven consecutive nodes were lengthened in concert, conduction velocity was dramatically decreased, eventually leading to failure of propagation at nodes with low excitability (Fig. 5f). While conduction speed decreased as more consecutive nodes were lengthened, the most significant change in propagation was introduced by modification of a single node (Fig. 5g; Supplemental Fig. 5). Together, these findings suggest that action potential propagation is highly tuned to the lengthening of individual nodes along the axon, with significant spike delays unavoidable when several nodes lengthen in response to learning.

Sheath addition following learning led to the closure of gaps in pre-existing myelin sheaths, generating new stretches of continuous myelination (see Figs. 2,3). To determine the effect of sheath addition on conduction velocity, we modeled consecutive sheath addition into an unmyelinated gap until the gap was eliminated (Fig. 5h). We used a gap length of 184 microns to represent the largest unmyelinated gap observed to be completely filled in with myelin (Supplemental Fig. 5). We found that up to three sheaths can be added to a single unmyelinated gap (Supplemental Fig. 5), therefore, we used these parameters to model the closure of gaps in myelination. Conduction speed increased exponentially with the addition of each new sheath, and the largest gain following the creation of a completely continuous stretch of myelin (Fig. 5i,j; Supplemental Fig. 5). Together, these results indicate that sheath retraction, resulting in increased node length, may slow or halt conduction along certain axons during the acquisition of a new motor task. In contrast, after motor learning, heightened generation of continuous myelination may speed up transmission.

\section{Behaviorally-activated axons show heightened myelin and nodal plasticity.}

Previous studies show that exogenous neuronal activity can drive oligodendrogenesis ${ }^{4}$ and myelination of specific axons ${ }^{12}$ in the adult brain. Yet, how myelination is modified by behavior on specific neuronal ensembles associated with learning or life experience remains unclear. To answer this question, we labeled behaviorallyactivated axons using an activity- and tamoxifen-dependent viral approach called Targeted Recombination in Active Populations (TRAP) $^{26,27}$. We combined this approach with longitudinal in vivo imaging to monitor the dynamic changes in myelin and nodes on activated neuronal ensembles involved in the forelimb reach. We injected L $2 / 3$ of the primary motor cortex of MOBP-EGFP;R26-lsltdTomato reporter mice with a cFos-ERT2-Cre-ERT2-PEST AAV, which drives tamoxifen-dependent Cre expression via the activitydependent $\mathrm{cFos}$ promoter ${ }^{28}$. Longitudinal in vivo imaging allowed us to identify neurons labeled by tamoxifen-intendent recombination

Figure 4 continued. d, Learning modulates the proportion of axons receiving heightened myelination in $L 1\left(F_{1,11.95}=12.65, p=0.0040\right)$. After learning, axons receive heightened myelination relative to before learning ( $p=0.0065$; Tukey's HSD) (d) and relative to axons in untrained mice ( $p=0.0087$; Tukey's HSD). e, Learning-induced sheath retraction precedes the post-learning burst in oligodendrogenesis in L1 (Student's $t$-test, $t(4.00)=-4.51$, $p=0.011)$. g, Learning modulates node lengthening in $L 2 / 3\left(F_{1,9}=15.85, p=0.0038\right)$. More nodes lengthen after than before learning in $L 2 / 3$ $(p=0.0014$; Tukey's HSD) and compared to untrained mice in $L 2 / 3$ ( $p=0.0009$; Tukey's HSD). $\mathbf{h}$, Learning modulates the proportion of axons with multiple dynamic nodes in $\mathrm{L} 2 / 3\left(\mathrm{~F}_{3,12}=9.87, \mathrm{p}=0.0015\right)$. In learning mice, significantly more axons with dynamic myelin possess multiple dynamic nodes than in untrained mice in L2/3 ( $p=0.015$; Tukey's HSD). i, Learning modulates the proportion of axons receiving heightened myelination in $L 2 / 3\left(F_{1,8.92}=7.87, p=0.021\right)$. After learning, axons receive heightened myelination relative to before learning $(p=0.0063)$ and relative to axons in untrained mice $(p=0.048$; Tukey's HSD). j, Learning-induced sheath retraction precedes the post-learning burst in oligodendrogenesis in L2/3 (Student's t-test, $\mathrm{t}(4.8)=-2.91, \mathrm{p}=0.035)$. ${ }^{*} \mathrm{p}<0.05,{ }^{* *} \mathrm{p}<0.01,{ }^{* * *} \mathrm{p}<0.0001$, NS, not significant; bars and error bars represent mean $\pm \mathrm{s} . \mathrm{e} . \mathrm{m}$. For detailed statistics, see Supplementary Table 3, Figure 4. 
bioRxiv preprint doi: https://doi.org/10.1101/2021.10.13.464319; this version posted October 15, 2021. The copyright holder for this preprint (which was not certified by peer review) is the author/funder. All rights reserved. No reuse allowed without permission.

a

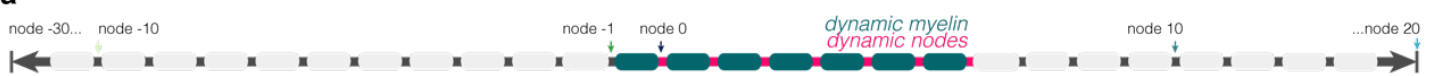

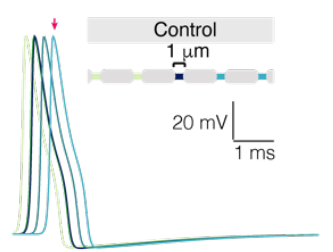

b

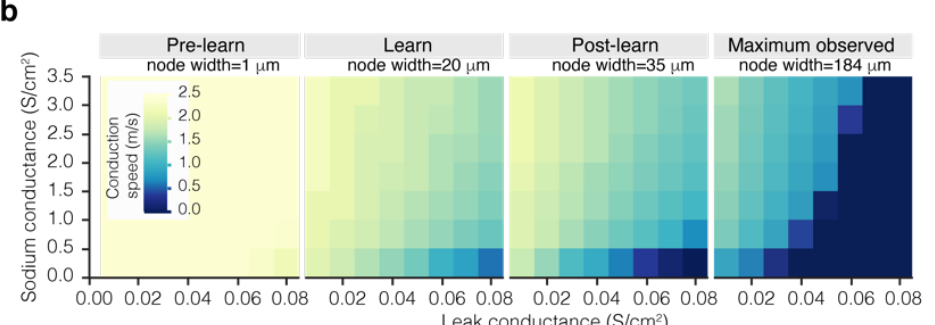

e

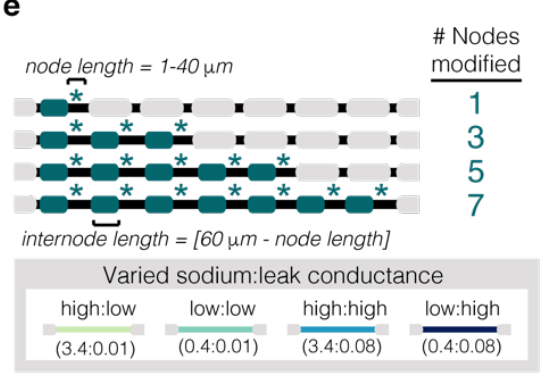

h

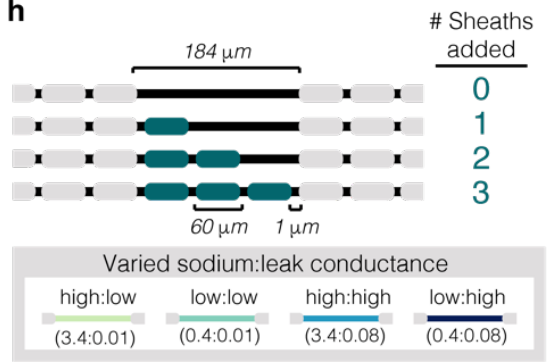

f i

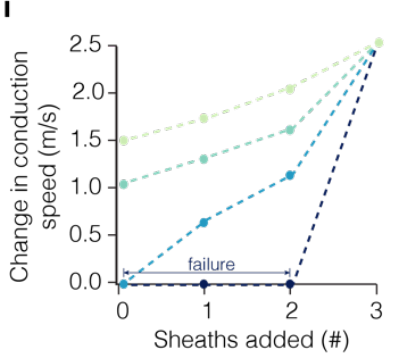

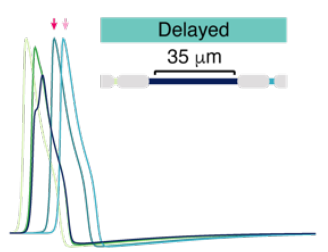

C

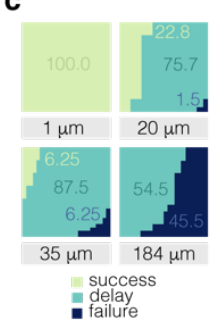

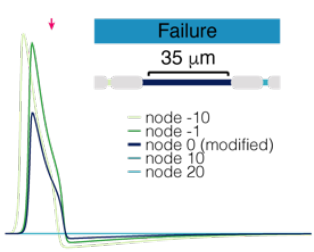

d

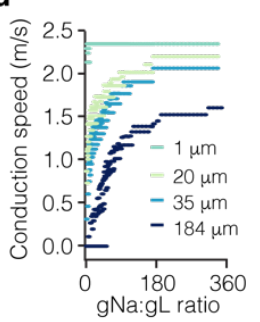

g

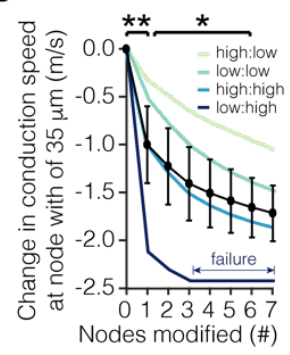

j

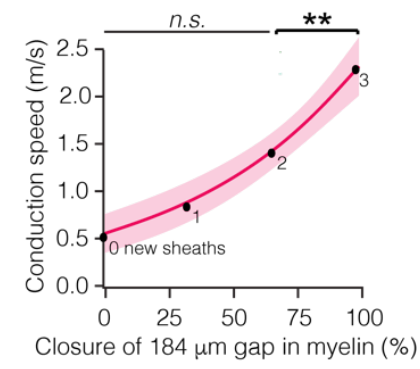

Figure 5 | Modeled effect of learning-induced sheath and nodal dynamics on conduction. a, Schematic of myelinated axon model. Control trace and example traces of three categories of propagation along the modified region of axon: no change in conduction speed ("Success"), slowed conduction speed ("Delayed"), and failure to propagate ("Failure"). b, Matrix of conduction speeds across node relative to the ratio of sodium:leak conductance. Node lengths are characteristic of lengths before learning ("Prelearn", 1 micron), directly after learning ("Learn", 20 microns), two weeks following learning ("Post-learn”, 35 microns), and of a large unmyelinated gap filled in by sheath addition ("Maximum observed", 184 microns). c, Proportion of events that result in successful (lime), delayed (teal), or failures (navy) in propagation at 1, 20,35, and 184 micron lengths. d, Modeled conduction speed as a function of sodium:leak conductance ratio at 1, 20,35, and 184 micron lengths. e, Schematization of model used to predict the effect of coupled node lengthening and sheath retraction on conduction speed. Nodes were lengthened incrementally from 1 to 40 microns and associated sheaths were retracted a reciprocal amount. Asterisks indicate node retraction, while blue sheaths indicate retraction. Nodes and sheaths were modified sequentially until 7 adjacent nodes were being lengthened at once. Four conductance ratios were modeled separately, using either $0.4 \mathrm{~S} / \mathrm{cm}^{2}$ or $3.4 \mathrm{~S} / \mathrm{cm}^{2}$ for sodium conductance $(\mathrm{gNa})$ and either $0.01 \mathrm{~S} / \mathrm{cm}^{2}$ or $0.08 \mathrm{~S} / \mathrm{cm}^{2}$ for leak conductance $(\mathrm{gL})$. $\mathbf{f}$, Modeled change in conduction speed as a function of node length at each of the four $\mathrm{gNa}$ : $\mathrm{gL}$ ratios with 1 (left) or 7 (right) nodes modified. g, Modeled conduction speed at a node length of 35 microns (dotted line in (f)) as a function of number of nodes modified. Learning modulates modeled conduction speed $\left(F_{7,21}=17.33, p<0.0001\right)$. Modification of 1 node significantly reduces conduction speed ( 0 vs. 1 nodes modified, $p=0.0008$; Tukey's HSD), as does modification of 6 nodes ( 1 vs. 6 nodes modified, $p=0.042$; Tukey's HSD). $\mathbf{h}$, Schematization of model used to predict the effect of adjacent sheath addition on conduction velocity. Sheaths of 60 microns in length were added sequentially to a 184 micron unmyelinated gap. i, Modeled change in conduction speed as a function of sheaths added. $\mathbf{j}$, Modeled conduction speed as a function of coverage of the unmyelinated gap. Sheath addition modulates conduction speed $\left(F_{3,9}=14.39, p=0.0009\right)$. The addition of a sheath that completely closes the unmyelinated gap results in a significant increase in conduction speed $(p=0.01) .{ }^{*} p<0.05,{ }^{* *} p<0.01,{ }^{* * *} p<0.0001$, NS, not significant; bars and error bars represent mean \pm s.e.m. For detailed statistics, see Supplementary Table 3, Figure 5.

and eliminate these false-positives from the behaviorally-activated condition. This approach revealed tamoxifen-independent tdTomato expression plateaued $\sim 40$ days following viral injection (Supplemental Fig. 6). Therefore, six weeks following viral 


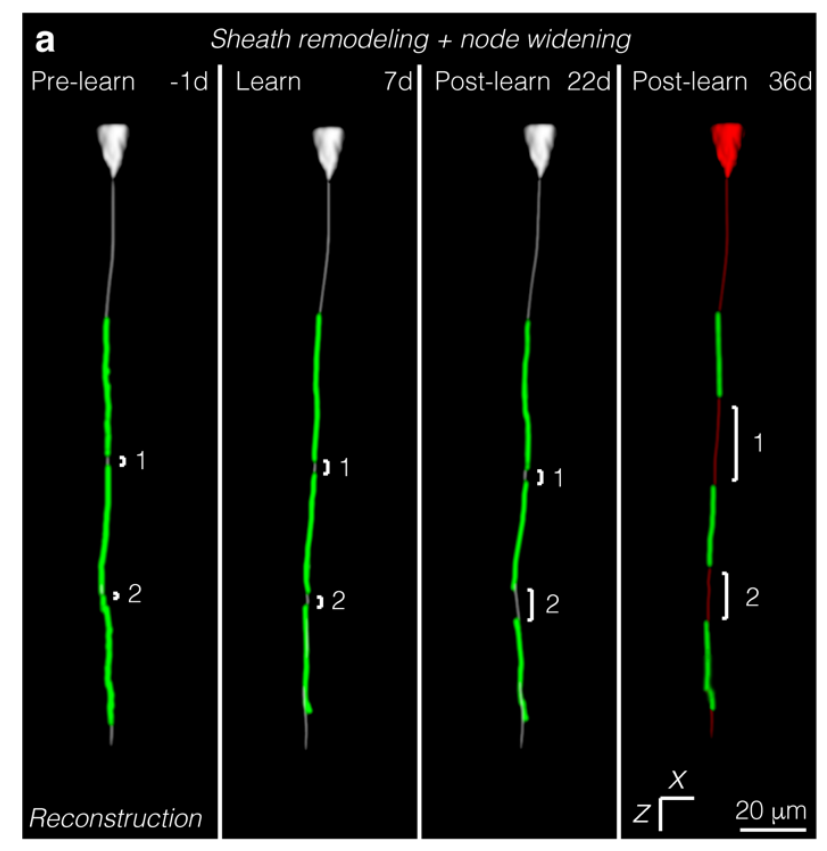

\section{b}

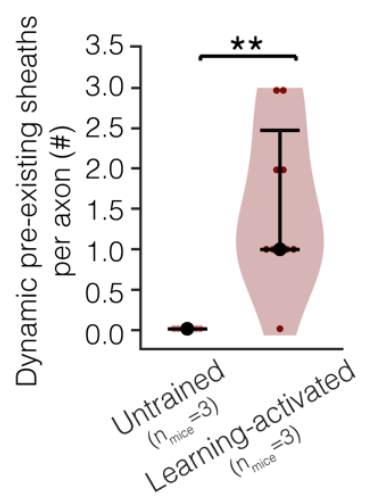

C
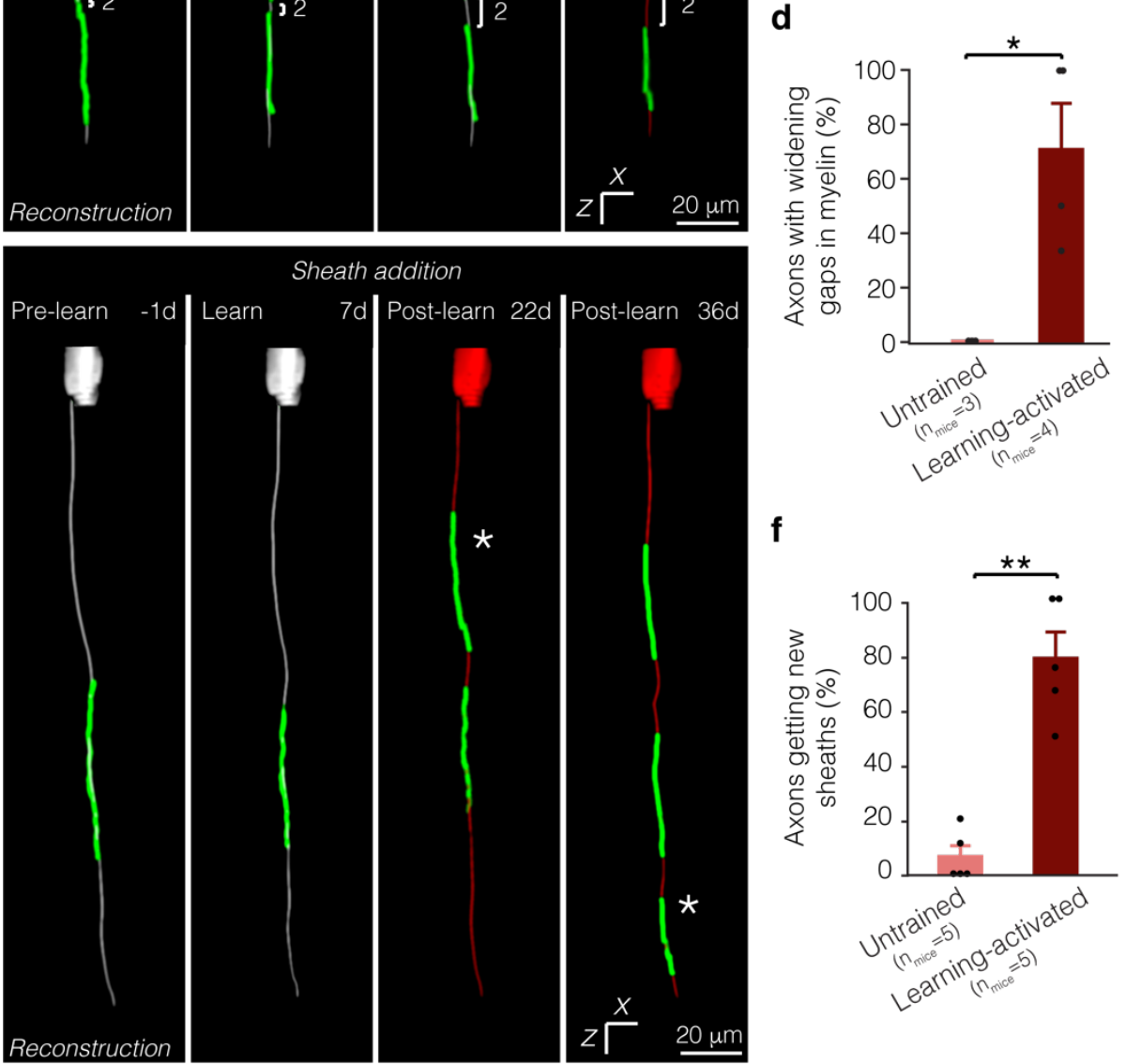

Sheath addition
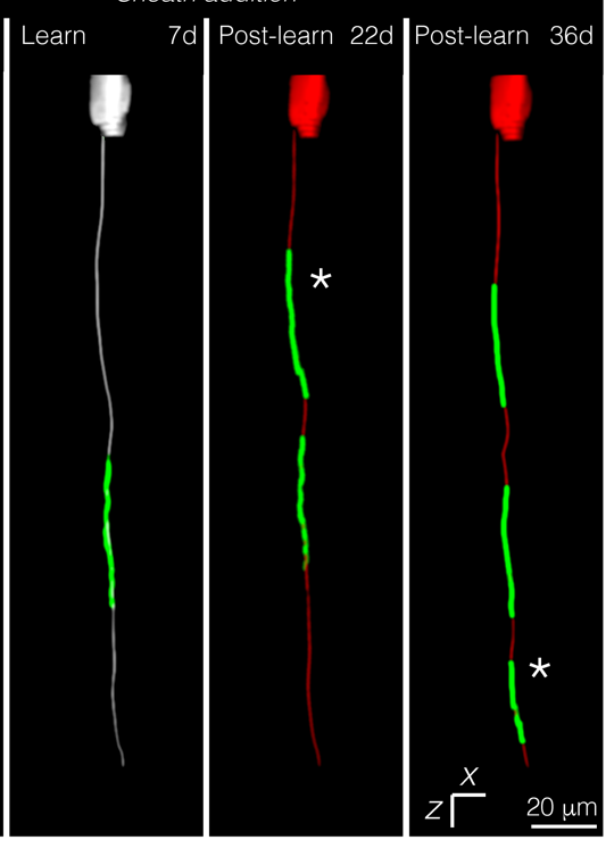

d

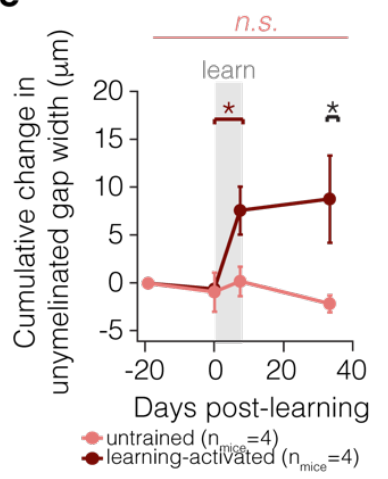

f

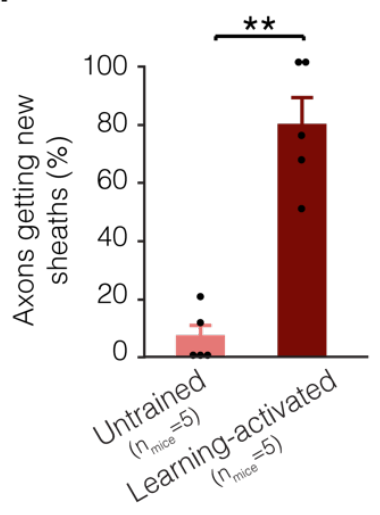

g
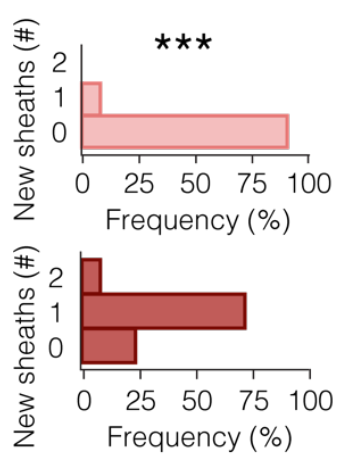

$\square$ untrained $\left(n_{\text {axons }}=25\right)$

$\square$ learning-activated $\left(\mathrm{n}_{\text {axons }}=13\right)$

Figure 6 | Learning-activated axons exhibit heightened myelin dynamics. a, Reconstructions of Layer 2/3 axons exhibiting coupled node lengthening and sheath retraction (top) and sheath addition (bottom) following learning. b, Learning-activated axons have significantly more sheath dynamics relative to non-activated axons (Student's t-test, $t(8.00)=-4.60, p=0.0017$ ). Dot and error bars represent median and I.Q.R. c, Learning modulates sheath retraction $\left(F_{3,115.8}=7.87, p<0.0001\right)$. During learning, sheaths on behaviorally-activated axons retract significantly ( $p=0.001$; Tukey's HSD), and one month after learning, sheaths are significantly shorter than those on axons in untrained mice ( $p=0.0002$; Tukey's HSD). $\mathbf{d}$, A significantly larger proportion of learning-activated axons have lengthened nodes relative to non-activated axons (Student's t-test, $t(3.00)=-4.12, p=0.026)$. e, Learning modulates node lengthening $\left(F_{3,18.45}=74.41, p=0.019\right)$. During learning, nodes on behaviorally-activated axons lengthen significantly $(p=0.046$; Tukey's HSD), and one month after learning, nodes are significantly longer than those on axons in untrained mice $(p=0.041$; Tukey's HSD). f, A significantly larger proportion of learning-activated axons receive sheaths after learning relative to non-activated axons (Student's $t$-test, $t(5.36)=-6.85, p=0.0008$ ). g, Learning-activated axons receive significantly more sheaths than non-activated axons following learning (Wilcoxon signed-rank; $p<0.0001$ ). ${ }^{*} p<0.05,{ }^{* *} p<0.01,{ }^{* * *} p<$ 0.0001 , NS, not significant; bars and error bars represent mean \pm s.e.m unless otherwise noted. For detailed statistics, see Supplementary Table 3, Figure 6.

injection, mice were trained on a forelimb reach task for seven days and 4-hydroxytamoxifen (4-OHT) or vehicle was delivered three hours after the final session of motor learning to label the behaviorally-activated neuron ensemble associated with the forelimb reach as previously described ${ }^{28}$. Importantly, we found that behaviorally-activated neurons and their associated axons were permanently labeled with tdTomato only in mice that received both motor learning and 4-OHT administration. Untrained mice that 
received 4-OHT or trained mice that received vehicle did not show a learning-associated increase in the number of neurons expressing tdTomato (Supplemental Fig. 6). The majority of behaviorallyactivated local neurons were found in Layer $2 / 3$ of cortex with descending projections (likely IT-type pyramidal neurons ${ }^{29}$ ) (Supplemental Fig. 6). Using this approach, we traced the proximal $240 \pm 86$ microns of the axon of behaviorally-activated neurons to determine changes in myelination of neuronal ensembles associated with motor learning (Fig. 6a; Supplemental Fig. 6).

We found that learning-activated axons showed increased numbers of retracting pre-existing myelin sheaths following motor learning compared to L2/3 tdTomato-positive neurons present at the onset of in vivo imaging in untrained mice (Fig. 6a,b). This sheath retraction was temporally correlated to learning, resulting in a significant decrease in sheath length during the learning period (Fig. 6c). These sheath dynamics resulted in increased numbers of lengthening unmyelinated gaps on learning-activated, sparsely myelinated axons relative to L2/3 axons in untrained mice (Fig. 6d). Similar to sheath retraction, gap lengthening was also temporally correlated with learning, and, on learning-activated axons, the gaps between myelin sheaths increased significantly, lengthening $8.32 \pm 2.38$ microns during the learning period and achieving significantly larger lengths than unmyelinated gaps in untrained mice by one month following learning (Fig. 6e). Next, we examined changes in the addition of new myelin sheaths on behaviorallyactivated axons. Following learning, a higher proportion of behaviorally-activated axons exhibited new sheath addition relative to axons in untrained mice $(78.33 \pm 21.73$ vs. $9.08 \pm 6.22 \%$ per mouse; Fig. 6f,g). These results suggest that altered sheath placement, rather than heightened oligodendrogenesis, drives increased sheath addition on behaviorally-activated axons in Layer $2 / 3$ of primary motor cortex following learning. Taken together, these data demonstrate that the diversity in patterns of intermittent myelination on cortical axons may be explained in part by activity-dependent recruitment of myelin sheaths and plasticity of pre-existing myelin and nodal distributions.

\section{Discussion}

Changes to nodal and myelin sheath properties are emerging as potential regulators of conduction, suggesting that experiencedriven changes to patterns of myelination may play a role in shaping neuronal communication during learning. Exploring learningdependent myelin dynamics on behaviorally-relevant axons is vital to understanding how changes to myelin might drive circuit dynamics during learning. Here, we establish that two forms of learning-related myelin plasticity-nodal dynamics and new sheath addition - are specifically targeted to motor cortical axons activated by motor learning in the healthy adult mouse. Learning-induced myelin plasticity proceeds in a staged response: during learning, preexisting sheath retraction creates new patterns of intermittent myelination on activated axons, while new sheaths from oligodendrocytes generated after learning preferentially eliminate large gaps in pre-existing myelin. Furthermore, we provide a biologically-relevant computational model which suggests that plastic changes to myelin pattern, nodal distribution, and ion channel properties along unmyelinated gaps may have profound effects on action potential propagation and circuit function.

Remodeling of pre-existing myelin sheaths was first proposed as a mechanism underlying the observation that adult-born oligodendrocytes generated shorter myelin segments than oligodendrocytes generated during development in the highly myelinated optic nerve ${ }^{30}$. Subsequent longitudinal in vivo imaging studies confirmed that pre-existing myelin sheaths could be remodeled and documented the dynamics of this process in the context of sensory deprivation or aging ${ }^{5,22,31}$. Yet, the link between changes in neuronal activity and the remodeling of pre-existing myelin sheaths remains unclear. Our studies indicate that myelin remodeling occurs specifically on myelinated axons with behaviorally-induced neuronal activity. These findings support previous work showing that generalized modifications to neuronal activity, such as changing sensory experience via monocular deprivation, modulate pre-existing myelin sheath length in a neuronal subclass-dependent manner ${ }^{22}$. During the formation of new myelin sheaths following oligodendrocyte generation, axonal vesicular release is key in regulating the growth of nascent sheaths ${ }^{10,11}$. This is controlled in part by modulating local calcium concentration within individual new myelin sheaths ${ }^{13,14}$. However, synaptic vesicle release regulates the growth of myelin sheath on certain glutamatergic neurons but not others ${ }^{32}$ suggesting neuronspecific or additional oligodendrocyte-intrinsic cues may play important roles in sheath formation. Indeed, oligodendrocyteintrinsic cues direct regional differences in myelin sheath lengths of cortical- versus spinal cord-derived oligodendrocytes ${ }^{33}$. These cues may include ion channel signaling via hyperpolarization-activated, cyclic nucleotide-gated ( $\mathrm{HCN})$ ion channels, and inhibitory transmembrane proteins such as Nogo-A, which modulate length of new myelin sheaths ${ }^{34,35}$. Future work investigating whether similar signaling mechanisms regulate changes in the length of pre-existing myelin sheaths on behaviorally-activated axons will provide additional insight into the fundamental biology regulating myelin remodeling.

Building evidence suggests that the patterns of myelination that emerge during development may be used to fine tune conduction properties of specific axons ${ }^{16}$. Furthermore, the varied distribution of lengths of nodes of Ranvier in the optic nerve and corpus callosum suggests that dynamically altering node parameters will alter conduction velocity ${ }^{17}$. Our data indicate that motor learning induces changes in node length, mediated through sheath retraction, specifically on the axons of behaviorally-activated neurons. How these dynamic changes modify ion channel composition of nodes of Ranvier or heminodes remains unknown. The distribution and composition of ion channels are destabilized following removal of myelin following demyelination leading to changes in axonal excitability (reviewed by Waxman ${ }^{36}$ ). Our computational model predicts that retraction of pre-existing myelin sheaths alters conduction speed across a range of sodium and leak conductances, suggesting that dynamic changes in myelination patterns may play an important role in cortical circuit function. Since assemblage of sodium and potassium channels localized to nodes varies depending on brain region, neuronal subclass, maturation, and myelination (reviewed by Suminaite et al. ${ }^{37}$ ), future work should consider these additional variables when exploring how learning-induced nodal dynamics alter axonal excitability and conduction.

The functional ramifications of learning-induced changes to myelination warrant further exploration. One possibility is that sheath dynamics during learning may serve to introduce variability in early stages of learning ${ }^{38}$. For example, altering spike-timing plasticity via changes in action potential conduction could modify circuit function. Although LTD and LTP can occur with pre-post spike delays of up to \pm 100 millisecond (ms), spike timing-dependent plasticity operates most strongly within a $10 \mathrm{~ms}$ window, with a sudden 1-5 ms transition between LTP and LTD ${ }^{39}$. This suggests that even small shifts in arrival time- - as predicted with alterations to node length or sheath addition - could be significant, changing not only the strength of the synaptic connection but also potentially determining whether a synapse is strengthened or weakened (see Fields ${ }^{40}$ ). Indeed, in the songbird forebrain and potentially in the rat neocortex, minor delays in conduction can influence the sequential activation of neuronal ensembles to profoundly shape communication within a circuit ${ }^{41}$. Here, we show that retraction of continuous myelin sheaths results in the generation of new areas of 
intermittent myelination during learning, and our modeling suggests this slows conduction during task acquisition. After learning, we find elimination of gaps in myelin by new sheaths to generate stretches of continuous myelination, resulting in an increase in conduction speed. Interestingly, the deposition of new myelin increases axon caliber during development in the peripheral nervous system ${ }^{42,43}$, potentially serving as an alternative mechanism to modulate conduction velocity. Together, our data indicate that the transition from partial to full myelination, and vice versa, may be important to dynamically alter neuronal communication when acquiring a new skill.

We previously reported that the generation of new oligodendrocytes in the forelimb motor cortex increases following the completion of reach task training ${ }^{20}$. Consistent with these results, here we found that new sheath addition via oligodendrogenesis increases after attaining proficiency in the reach task suggesting this process may perform a distinct role in the late phases of learning. Recent work indicates that the generation of new oligodendrocytes regulates memory consolidation ${ }^{8,9}$. Our computational modeling suggests new sheath addition may serve to speed up conduction along consolidated neural circuits after learning. However, it is important to consider that myelin serves additional roles beyond influencing conduction speed, including metabolic support of axons $^{44,45}$. Recent studies suggest that oligodendroglial metabolic support of axons contributes to information processing ${ }^{46}$. The high density of connections in the cortex results in substantial energetic demands ${ }^{47}$ and activity-dependent myelin sheath addition may reflect increased metabolic needs due to heightened neuronal activity. Previous studies show that lactate, an oligodendrocytederived source of metabolic support to axons ${ }^{44,45,48}$, can rescue functional decline in the optic nerve due to glycogen depletion ${ }^{49}$. Furthermore, since myelin acts as a barrier to accessing extracellular energy supplies ${ }^{50,51}$, removal of myelin via sheath retraction may be driven by metabolic need, exposing axonal membrane to allow access to extracellular glucose. Exploring the effects of learninginduced myelin dynamics in the context of neuronal metabolic demands will improve our understanding of the roles of oligodendrocytes and myelination in information processing.

Another possible role for learning-induced nodal and myelin dynamics is to regulate the plasticity of axon morphology and synaptic connections. On PV interneurons, the degree of myelination is correlated to both branch morphology and the number of en passante boutons ${ }^{52}$. Furthermore, on intermittently myelinated L2/3 pyramidal neurons, unmyelinated regions contain both afferent and efferent synapses ${ }^{2}$. The staged response of learning-related myelin dynamics, where the removal of existing myelin and suppression of oligodendrogenesis occurs during learning ${ }^{20}$, may enable the formation of new synaptic connections in motor cortex. Subsequently, targeted sheath addition following learning could lead to the closure of a learning-related window of heightened plasticity, as suggested in studies modulating the duration of sensory map plasticity using Nogo receptor (NgR) knockout mice ${ }^{53}$. While exogenous stimulation of parvalbuminpositive axons drives newly-generated axonal collaterals that receive de novo myelination ${ }^{54}$, recent work shows pre-existing myelin dynamics are independent of increased axon branching events in visual cortex following monocular deprivation ${ }^{22}$. Additional information examining the specific timing of myelin dynamics and neuronal morphological plasticity will aid in determining direct and indirect effects of these processes.

Exploring the link between behaviorally-relevant neuronal activity and changes in myelination has important implications for myelin repair in the context of neurological disease. In multiple sclerosis, disability is lessened as remyelination increases ${ }^{55}$, but the extent of remyelination of lesions can differ within the same individual $^{56,57}$. Furthermore, in regions of continuous myelination, the pattern of myelination is often re-established following acute demyelination, while regions of discontinuous myelination are less likely to be replaced ${ }^{58,59}$. Together, these data suggest that lesions comprising different neuronal circuits may receive different levels of myelin repair. Here, we show that new myelin sheath addition and changes to pre-existing myelin sheath length are targeted to axons in a behaviorally-activated circuit. Our work establishes that behavior shapes the pattern of myelination on the level of individual axons within defined, activated circuits in the healthy brain. These findings raise the possibility that behaviorally-induced neuronal activity may modulate the specificity and efficacy of myelin repair following a demyelinating injury. Understanding how and when the brain engages in plastic changes to myelin could inform interventions to address myelin-altering pathology, as seen in multiple sclerosis and stroke, where targeted myelin dynamics hold potential to improve therapeutic success.

\section{Acknowledgements}

We thank Mike Hall for machining expertise; Helena Barr for the conversation that re-initiated interest in labeling activated axons; and past and current members of the Hughes and Welle labs for discussions.

\section{Funding}

MAT is supported by the NIH Institutional Neuroscience Graduate Training Grant (5T32NS099042-17). Funding was provided by the Boettcher Foundation Webb-Waring Biomedical Research Award, the Whitehall Foundation, and the National Multiple Sclerosis Society (RG-1701-26733) and NINDS (NS115975) to EGH.

\section{Author Contributions}

EGH and CMB conceived the project. CMB designed and performed experiments, analyzed data, and generated all figures. RH performed all viral injections and performed surgeries. MAT performed surgeries. LC performed additional imaging analyses. AC provided technical support for all mouse lines. APP supervised the computational modulating and EGH supervised the project. $\mathrm{CMB}$ and EGH wrote the manuscript with input from other authors.

\section{Competing Interests}

The authors declare no competing financial interests.

\section{Data and materials availability}

All data that support the findings, tools and reagents will be shared on an unrestricted basis; requests should be directed to the corresponding author.

\section{List of Supplementary Materials}

Supplementary Tables 1 to 3

Supplementary Figs. 1 to 6

\section{References}

1. Lillie, R. S. FACTORS AFFECTING TRANSMISSION AND RECOVERY IN THE PASSIVE IRON NERVE MODEL. Journal of General Physiology 7, 473-507 (1925).

2. Tomassy, G. S. et al. Distinct Profiles of Myelin Distribution Along Single Axons of Pyramidal Neurons in the Neocortex. Science 344, 319-324 (2014).

3. Yakovlev, P. \& Lecours, A. The myelogenetic cycles of regional maturation of the brain. Regional development of the brain in early life. Edited by: Minkowski A. 1967. (Oxford: Blackwell Scientific Publications, 1967).

4. Gibson, E. M. et al. Neuronal Activity Promotes Oligodendrogenesis and Adaptive Myelination in the Mammalian Brain. Science 344, 1252304 (2014). 
5. Hughes, E. G., Orthmann-Murphy, J. L., Langseth, A. J. \& Bergles, D. E. Myelin remodeling through experience-dependent oligodendrogenesis in the adult somatosensory cortex. Nature Neuroscience 21, 696-706 (2018).

6. McKenzie, I. A. et al. Motor skill learning requires active central myelination. Science 346, 318-322 (2014).

7. Wang, F. et al. Myelin degeneration and diminished myelin renewal contribute to age-related deficits in memory. Nature Neuroscience 23, 481-486 (2020).

8. Pan, S., Mayoral, S. R., Choi, H. S., Chan, J. R. \& Kheirbek, M. A. Preservation of a remote fear memory requires new myelin formation. Nature Neuroscience 23, 487-499 (2020).

9. Steadman, P. E. et al. Disruption of Oligodendrogenesis Impairs Memory Consolidation in Adult Mice. Neuron S0896627319308864 (2019) doi:10.1016/j.neuron.2019.10.013.

10. Hines, J. H., Ravanelli, A. M., Schwindt, R., Scott, E. K. \& Appel, B. Neuronal activity biases axon selection for myelination in vivo. Nat Neurosci 18, 683-689 (2015).

11. Mensch, S. et al. Synaptic vesicle release regulates myelin sheath number of individual oligodendrocytes in vivo. Nat Neurosci $\mathbf{1 8}$, 628-630 (2015).

12. Mitew, S. et al. Pharmacogenetic stimulation of neuronal activity increases myelination in an axon-specific manner. Nature Communications 9, 306 (2018).

13. Baraban, M., Koudelka, S. \& Lyons, D. A. Ca $2+$ activity signatures of myelin sheath formation and growth in vivo. Nature Neuroscience 21, 19-23 (2018).

14. Krasnow, A. M., Ford, M. C., Valdivia, L. E., Wilson, S. W. \& Attwell, D. Regulation of developing myelin sheath elongation by oligodendrocyte calcium transients in vivo. Nature Neuroscience 21, 24-28 (2018).

15. Seidl, A. H., Rubel, E. W. \& Harris, D. M. Mechanisms for Adjusting Interaural Time Differences to Achieve Binaural Coincidence Detection. J. Neurosci. 30, 70-80 (2010).

16. Ford, M. C. et al. Tuning of Ranvier node and internode properties in myelinated axons to adjust action potential timing. Nature Communications 6, 8073 (2015).

17. Arancibia-Cárcamo, I. L. et al. Node of Ranvier length as a potential regulator of myelinated axon conduction speed. eLife Sciences 6, e23329 (2017).

18. Salami, M., Itami, C., Tsumoto, T. \& Kimura, F. Change of conduction velocity by regional myelination yields constant latency irrespective of distance between thalamus and cortex. PNAS 100, 6174-6179 (2003).

19. Auer, F., Vagionitis, S. \& Czopka, T. Evidence for Myelin Sheath Remodeling in the CNS Revealed by In Vivo Imaging. Current Biology 28, 549-559.e3 (2018).

20. Bacmeister, C. M. et al. Motor learning promotes remyelination via new and surviving oligodendrocytes. Nature Neuroscience $\mathbf{2 3}$, 819-831 (2020).

21. Cullen, C. L. et al. Periaxonal and nodal plasticities modulate action potential conduction in the adult mouse brain. Cell Reports 34, 108641 (2021).

22. Yang, S. M., Michel, K., Jokhi, V., Nedivi, E. \& Arlotta, P. Neuron class-specific responses govern adaptive myelin remodeling in the neocortex. Science 370, (2020).

23. Bishop, D. et al. Near-infrared branding efficiently correlates light and electron microscopy. Nat Methods 8, 568-570 (2011).

24. Etxeberria, A. et al. Dynamic Modulation of Myelination in Response to Visual Stimuli Alters Optic Nerve Conduction Velocity. J. Neurosci. 36, 6937-6948 (2016).

25. Coggan, J. S., Prescott, S. A., Bartol, T. M. \& Sejnowski, T. J. Imbalance of ionic conductances contributes to diverse symptoms of demyelination. PNAS 107, 20602-20609 (2010).

26. Guenthner, C. J., Miyamichi, K., Yang, H. H., Heller, H. C. \& Luo, L. Permanent Genetic Access to Transiently Active Neurons via TRAP: Targeted Recombination in Active Populations. Neuron 78, 773-784 (2013).

27. DeNardo, L. A. et al. Temporal evolution of cortical ensembles promoting remote memory retrieval. Nature Neuroscience $\mathbf{2 2}$, 460-469 (2019).

28. Ye, L. et al. Wiring and Molecular Features of Prefrontal Ensembles Representing Distinct Experiences. Cell 165, 1776 1788 (2016).
29. Papale, A. E. \& Hooks, B. M. Circuit Changes in Motor Cortex During Motor Skill Learning. Neuroscience 368, 283-297 (2018).

30. Young, K. M. et al. Oligodendrocyte Dynamics in the Healthy Adult CNS: Evidence for Myelin Remodeling. Neuron 77, 873885 (2013).

31. Hill, R. A., Li, A. M. \& Grutzendler, J. Lifelong cortical myelin plasticity and age-related degeneration in the live mammalian brain. Nature Neuroscience 21, 683-695 (2018).

32. Koudelka, S. et al. Individual Neuronal Subtypes Exhibit Diversity in CNS Myelination Mediated by Synaptic Vesicle Release. Current Biology 26, 1447-1455 (2016).

33. Bechler, M. E., Byrne, L. \& ffrench-Constant, C. CNS Myelin Sheath Lengths Are an Intrinsic Property of Oligodendrocytes. Current Biology 25, 2411-2416 (2015).

34. Swire, M. et al. Oligodendrocyte HCN2 Channels Regulate Myelin Sheath Length. J. Neurosci. 41, 7954-7964 (2021).

35. Chong, S. Y. C. et al. Neurite outgrowth inhibitor Nogo-A establishes spatial segregation and extent of oligodendrocyte myelination. PNAS 109, 1299-1304 (2012).

36. Waxman, S. G. Axonal conduction and injury in multiple sclerosis: the role of sodium channels. Nat Rev Neurosci 7, 932941 (2006).

37. Suminaite, D., Lyons, D. A. \& Livesey, M. R. Myelinated axon physiology and regulation of neural circuit function. Glia 67 , 2050-2062 (2019).

38. Dhawale, A. K., Smith, M. A. \& Ölveczky, B. P. The Role of Variability in Motor Learning. Annual Review of Neuroscience 40, 479-498 (2017).

39. Feldman, D. E. The Spike-Timing Dependence of Plasticity. Neuron 75, 556-571 (2012).

40. Fields, R. D. A new mechanism of nervous system plasticity: activity-dependent myelination. Nat Rev Neurosci 16, 756-767 (2015).

41. Egger, R. et al. Local Axonal Conduction Shapes the Spatiotemporal Properties of Neural Sequences. Cell 183, 537548.e12 (2020).

42. de Waegh, S. M., Lee, V. M.-Y. \& Brady, S. T. Local modulation of neurofilament phosphorylation, axonal caliber, and slow axonal transport by myelinating Schwann cells. Cell 68, 451-463 (1992).

43. Garcia, M. L. et al. NF-M is an essential target for the myelindirected "outside-in" signaling cascade that mediates radial axonal growth. Journal of Cell Biology 163, 1011-1020 (2003).

44. Fünfschilling, U. et al. Glycolytic oligodendrocytes maintain myelin and long-term axonal integrity. Nature 485, 517-521 (2012).

45. Lee, Y.et al. Oligodendroglia metabolically support axons and contribute to neurodegeneration. Nature 487, 443-448 (2012).

46. Moore, S. et al. A role of oligodendrocytes in information processing. Nat Commun 11, 5497 (2020).

47. Harris, J. J. \& Attwell, D. The Energetics of CNS White Matter. $J$. Neurosci. 32, 356-371 (2012).

48. Meyer, N. et al. Oligodendrocytes in the Mouse Corpus Callosum Maintain Axonal Function by Delivery of Glucose. Cell Reports 22, 2383-2394 (2018).

49. Brown, A. M., Tekkök, S. B. \& Ransom, B. R. Glycogen Regulation and Functional Role in Mouse White Matter. The Journal of Physiology 549, 501-512 (2003).

50. Trevisiol, A. et al. Structural myelin defects are associated with low axonal ATP levels but rapid recovery from energy deprivation in a mouse model of spastic paraplegia. PLOS Biology 18, e3000943 (2020).

51. Chamberlain, K. A. \& Sheng, Z.-H. Mechanisms for the maintenance and regulation of axonal energy supply. Journal of Neuroscience Research 97, 897-913 (2019).

52. Stedehouder, J. et al. Fast-spiking Parvalbumin Interneurons are Frequently Myelinated in the Cerebral Cortex of Mice and Humans. Cereb Cortex 27, 5001-5013 (2017).

53. Kalish, B. T. et al. Single-nucleus RNA sequencing of mouse auditory cortex reveals critical period triggers and brakes. PNAS 117, 11744-11752 (2020).

54. Stedehouder, J., Brizee, D., Shpak, G. \& Kushner, S. A. ActivityDependent Myelination of Parvalbumin Interneurons Mediated by Axonal Morphological Plasticity. J. Neurosci. 38, 3631-3642 (2018). 
bioRxiv preprint doi: https://doi.org/10.1101/2021.10.13.464319; this version posted October 15, 2021. The copyright holder for this preprint (which was not certified by peer review) is the author/funder. All rights reserved. No reuse allowed without permission.

55. Bramow, S. et al. Demyelination versus remyelination in progressive multiple sclerosis. Brain 133, 2983-2998 (2010).

56. Patrikios, P. et al. Remyelination is extensive in a subset of multiple sclerosis patients. Brain 129, 3165-3172 (2006).

57. Goldschmidt, T., Antel, J., König, F. B., Brück, W. \& Kuhlmann, T. Remyelination capacity of the MS brain decreases with disease chronicity. Neurology 72, 1914-1921 (2009).
58. Snaidero, N. et al. Myelin replacement triggered by single-cell demyelination in mouse cortex. Nat Commun 11, 4901 (2020).

59. Orthmann-Murphy, J. et al. Remyelination alters the pattern of myelin in the cerebral cortex. eLife 9, e56621 (2020). 


\section{Methods}

Animals. All animal experiments were conducted in accordance with protocols approved by the Animal Care and Use Committee at the University of Colorado Anschutz Medical Campus. Male and female mice used in these experiments were kept on a $14 \mathrm{~h}$ light/10h dark schedule with ad libitum access to water and food, except during trainingrelated food restriction (see Forelimb Reach Training). Mice were randomly assigned to experimental groups and were precisely age-matched across groups. R26-lsl-tdTomato reporter mice (Ai9; JAX: 007909), congenic C57BL/6N $M O B P-E G F P(\mathrm{MGI}: 4847238)$ lines, which have been previously described ${ }^{\mathbf{5 , 2 0}}$, were used for two-photon imaging.

Two-photon microscopy. Cranial windows were prepared as previously described ${ }^{\mathbf{6 0}}$. Induction of anesthesia in six- to eightweek-old mice was accomplished with isoflurane inhalation (induction, $5 \%$; maintenance, $1.5-2.0 \%$, mixed with $0.5 \mathrm{~L} / \mathrm{min}$ O2), and mice were kept at $37^{\circ} \mathrm{C}$ body temperature with a thermostat-controlled heating plate. The skin over the right cerebral hemisphere was removed, the skull was cleaned, and a $2 \times 2 \mathrm{~mm}$ region of skull centered over the forelimb region of the primary motor cortex ( 0 to $2 \mathrm{~mm}$ anterior to bregma and 0.5 to $2.5 \mathrm{~mm}$ lateral) was opened using a high-speed dental drill. A piece of cover glass (VWR, No. 1) was then placed in the craniotomy and sealed with Vetbond (3M) followed by dental cement (C\&B Metabond). Carprofen (5mg/kg) was administered subcutaneously prior to awakening and for three additional days for analgesia. To stabilize the head during imaging, a custom metal plate with a central hole was attached to the skull. In vivo imaging sessions began 2-3 weeks postsurgery and took place once per week. At each imaging time point, mice were anesthetized with isoflurane and immobilized by attaching the head plate to a custom stage. Images were collected using a Zeiss LSM 7MP microscope equipped with a BiG GaAsP detector using a mode-locked Ti:sapphire laser (Coherent Ultra) tuned to $920 \mathrm{~nm}$. The average power at the sample during imaging was $5-30 \mathrm{~mW}$. Vascular and cellular landmarks were used to identify the same cortical area over longitudinal imaging sessions. Image stacks were acquired with a Zeiss W "Plan-Apochromat" 20X/1.0 NA water immersion objective giving a volume of $425 \mu \mathrm{m} \times 425 \mu \mathrm{m} \times 336 \mu \mathrm{m}(2,048 \times 2,048$ pixels, $1.5 \mu \mathrm{m}$ step size; corresponding to layers I-III, $0-336 \mu \mathrm{m}$ from the meninges, or layer $2 / 3110-446 \mu \mathrm{m}$ from the meninges).

Forelimb reach training . Mice were weighed, habituated to a training box for 20 minutes, and deprived of food 24 hours prior to training. The training box was fitted with a window providing access to a pellet located on a shelf $1 \mathrm{~cm}$ anterior and $1 \mathrm{~mm}$ lateral to the right-hand side of the window. After one session of initial habituation, training sessions began daily for 20 minutes or 20 successful reaches, whoever occurred first. Mice learned to reach for the pellet using their left hand. Successes were counted when the mouse successfully grabbed the pellet and transported it inside the box. Errors were qualified in three ways: "Reach error" (the mouse extends its paw out the window but does not grab the pellet), "Grasp error" (the mouse reaches the pellet but does not successfully grasp onto it), and "Retrieval error" (the mouse grasps the pellet but does not succeed in returning it to the box). Mice were kept on a restricted diet throughout training to maintain food motivation but were weighed daily to ensure weight loss did not exceed $10 \%$. For forelimb reach training, mice underwent habituation (average of $\sim 1$ days of exposure) followed by training until seven consecutive days of training with reach attempts were recorded. Similar to previously published findings, over $90 \%$ of mice trained in forelimb reach context were able to learn the task ${ }^{61}$; mice were excluded if they were not able on a single day to succeed in at least $10 \%$ of reaches (Supplementary Fig. 2). To control for any experimenter effects in forelimb reach training results, all mice were trained by the same experimenter (i.e. control and experimental mice were only compared if trained by the same experimenter).

Labeling of behaviorally-activated axons. $R 26$-lsl-tdTomato or MOBP-EGFP;R26-lsl-tdTomato reporter mice were injected with 1ul of AAV-8-cFos-ERT2-Cre-ERT2-PEST ${ }^{28}$ in which CreER expression is driven by the activity-dependent $\operatorname{Arc}$ promoter at a 1:8 dilution (stock virus titer: $8.8 \times 10^{12}$ ). 6 weeks later, mice were trained in a forelimb reach task (see Forelimb reach training). Age-matched controls were exposed to the box, fasted, and injected with tamoxifen but not given reach training. $10 \mathrm{mg} / \mathrm{kg}$ of 4-OHT was given to all mice three hours after the last training session to enable CreER-mediated recombination. Mice were then imaged for an additional 4 weeks following tamoxifen to allow the full expression of tdTomato fluorophores (see Supplementary Fig. 6).

Near-infrared branding. Near-infrared branding (NIRB) marks were generated using our two-photon microscope (described above in Two-photon microscopy). The orientation and size of NIRB marks was regulated by modulating laser power and exposure time, as described previously (Bishop et al., 2011). To generate NIRB marks, we used a Zeiss W "PlanApochromat" 20X/1.0 NA water immersion objective. To locate cells and structures of interest within the longitudinallyimaged window, we burned a $\sim 500 \mu \mathrm{m} \times 500 \mu \mathrm{m}$ box with asymmetrical markings, ensuring burn marks were $100 \mu \mathrm{m}$ away from the area of interest (Supplementary Fig. 1,4). We then captured a low-resolution two-photon image stack of the completed NIRB marks and their relationship to longitudinally-imaged structures to locate them across in vivo images and in post-hoc immunostained sections, using emission filters for yellow-green fluorescent proteins and DsRed. Prior to marking the brain with NIRB, mice were anesthetized with an intraperitoneal injection of sodium pentobarbital $(100 \mathrm{mg} / \mathrm{kg}$ b.w.) and transcardially perfused with $4 \%$ paraformaldehyde in $0.1 \mathrm{M}$ phosphate buffer $(\mathrm{pH} 7.0$ 7.4).

Correlated in vivo and immunohistochemistry. Brains were postfixed in $4 \%$ PFA for $1 \mathrm{hr}$ at $4{ }^{\circ} \mathrm{C}$, transferred to $30 \%$ sucrose solution in PBS (pH 7.4), and stored at $4{ }^{\circ} \mathrm{C}$ for at least $24 \mathrm{~h}$. Brains were extracted, frozen in TissuePlus O.C.T, and sectioned at $30 \mu \mathrm{m}$ thick. To obtain correlated images of immuno-processed sections, we first sectioned out the cranial window area and oriented the tissue to be cryosectioned in a plane parallel to the surface of the imaged area of the brain. Once sectioned, we selected fixed cryosections to immunostain by locating the NIRB markings on a confocal microscope (Zeiss LSM 510). 
Immunostaining was performed on free-floating sections. Sections were pre-incubated in blocking solution $5 \%$ normal donkey serum, $2 \%$ bovine g-globulin, $0.3 \%$ Triton $\mathrm{X}-100$ in PBS, $\mathrm{pH}$ 7.4) for $1-4 \mathrm{~h}$ at room temperature, then incubated overnight at $4{ }^{\circ} \mathrm{C}$ in primary antibody (listed along with secondary antibodies in Supplementary Table 2). Secondary antibody incubation was performed at room temperature for 2 h. Sections were mounted on slides with Vectashield antifade reagent (Vector Laboratories). Images were acquired with a laser-scanning confocal microscope (Zeiss LSM 510).

Image processing and analysis. Image stacks and time-series were analyzed using FIJI/ImageJ. All analysis was performed on images, which were pre-processed by with a Gaussian blur filter (radius $=1$ pixels) to denoise and allow identification of individual myelin sheaths and nodes. When generating figures, image brightness and contrast levels were adjusted for clarity. Longitudinal image stacks were registered using FIJI plugins 'Correct 3D drift'67 or 'PoorMan3DReg'. When possible, blinding to experimental condition was used in analyzing image stacks from two-photon imaging.

Myelin sheath and node analysis and analysis of myelin profiles along single axons. In vivo z-stacks were collected from $M O B P-E G F P$ mice using two-photon microscopy. Zstacks were processed with a 1-pixel Gaussian blur filter to aid in the identification of myelin internodes. Myelin paranodes and nodes of Ranvier were identified as described previously (Hughes et al., 2018), by increase in fluorescence intensity for paranodes and a decrease to zero in EGFP fluorescence intensity for nodes. Node length was defined as the distance between each paranodes as measured by Simple Neurite Tracer. Two-color images of tdTom + axons and their associated myelin sheaths (labeled with EGFP) were registered using FIJI plugins 'Correct 3D drift' or 'PoorMan3DReg'. Axons, myelin, and nodes were then traced using Simple Neurite Tracer at baseline, the first day of training, the last day of training, and either 2 or 4 weeks following training. Cells and sheaths that resided within a volume of $425 \times 425 \times 110 \mu \mathrm{m}^{3}$ from the pial surface were considered in Layer 1 and those at a depth of $110-446 \mu \mathrm{m}^{2}$ were considered Layer $2 / 3$. Sheaths were defined as new or pre-existing. Persisting sheaths and nodes lasted for the entire imaging time course; new sheaths appeared after day 0 .

Computational modeling. We used the NEURON simulation environment (v.7.4; Hines \& Carnevale, 1997) to simulate action potential propagation along a myelinated axon. Details of the parameters used are summarized in Supplemental Table 1. The axon is divided into compartments representing the node and internode. 52 nodes and 51 myelin internodes were simulated, and conduction speed was measured between the $30^{\text {th }}$ and $40^{\text {th }}$ node.

Spatial resolution was set using 5 segments per cellular compartment, which ranged from 1-184 microns in length. Parameter bounds are described in Supplemental Table 1. Briefly, the lower and upper bounds for sodium conductance and leak conductance were defined using biologically relevant values consistent with previous publications ${ }^{25}$. Myelinated axon and node diameters were set to 0.902 microns and 0.72 microns, respectively, and were within the range of experimentally-derived values for myelinated cortical axons from previous publications ${ }^{17,58,62}$. Pre-learning (1 micron), learning (20 microns), and post-learning (35 microns) node lengths were set using experimentally-measured values (see Figs. 1-3; Supplemental Fig. 5). Similarly, myelin sheath length was set to 60 microns using experimentally-derived values (see Supplemental Fig. 5). A g-ratio of 0.7 was used, consistent with values determined from previous publications ${ }^{58,62}$. Temperature was set to $37^{\circ} \mathrm{C}$, as in previous publications $^{17,63}$. Hodgkin-Huxley style kinetics ${ }^{64,65}$ for NaV1.6 channels were fit using data from Huguenard et al. ${ }^{66}$ and Hamill et al ${ }^{67}$ and $\mathrm{Kv} 1$ channel kinetics were fit based on rates from Hamill et al. ${ }^{67}$. Model files and equations were obtained from $\mathrm{Hu}$ et al. ${ }^{63}$.

Statistics. A detailed and complete report of all statistics used, including definitions of individual measures, summary values, sample sizes, and all test results can be found in Supplementary Table 3. Sample sizes were not predetermined using statistical methods, but are comparable to relevant publications. All data were initially screened for outliers using IQR methods. All mice in a litter underwent cranial window surgery and concurrent two-photon imaging and training timelines were designated to be a "batch". When possible, experimental groups were replicated in multiple batches with multiple experimental groups per batch. However, due to the longitudinal nature of our study, and the clarity of cranial windows used to conduct two-photon imaging, we could not predict which mice would produce full datasets when assigning them to experimental conditions. As a result, not all batches contain all experimental groups. Statistical analyses were conducted using JMP (SAS). We first assessed normality in all datasets using the Shapiro-Wilk test. When normality was violated, we used non-parametric rank-sum tests. When normality was satisfied, we used parametric statistics including paired or unpaired two-tailed Student's t-tests (depending on within- or between-subjects nature of analysis), or one-way and two-way ANOVA with Tukey's HSD post-hoc tests. Twotailed tests and $\alpha \leq 0.05$ were always employed unless otherwise specified. For statistical mixed modelling, we used a restricted maximum likelihood (REML) approach with unbounded variance component, and least-squared Tukey's HSD post-hoc tests. All models were conducted with either one or two fixed effects, in which case we ran full factorial models. For all models, we used "Mouse ID" as a random variable unless otherwise specified. Where we found significant effects, we subsequently calculated effect size using Cohen's d, and these results may be found in Supplementary Table 3. For data visualization, all error bars represent the standard error of the mean unless otherwise specified.

\section{Methods references}

60. Hughes, E. G., Kang, S. H., Fukaya, M. \& Bergles, D. E. Oligodendrocyte progenitors balance growth with selfrepulsion to achieve homeostasis in the adult brain. Nat Neurosci 16, 668-676 (2013).

61. Xu, T. et al. Rapid formation and selective stabilization of synapses for enduring motor memories. Nature 462, 915919 (2009). 
bioRxiv preprint doi: https://doi.org/10.1101/2021.10.13.464319; this version posted October 15, 2021. The copyright holder for this preprint (which was not certified by peer review) is the author/funder. All rights reserved. No reuse allowed without permission.

62. Cohen, C. C. H. et al. Saltatory Conduction along Myelinated Axons Involves a Periaxonal Nanocircuit. Cell 180, 311-322.e15 (2020).

63. Hu, W. et al. Distinct contributions of Nav1.6 and Nav1.2 in action potential initiation and backpropagation. Nat Neurosci 12, 996-1002 (2009).

64. Mainen, Z. F., Joerges, J., Huguenard, J. R. \& Sejnowski, T. $\mathrm{J}$. A model of spike initiation in neocortical pyramidal neurons. Neuron 15, 1427-1439 (1995).

65. Hodgkin, A. L. \& Huxley, A. F. A quantitative description of membrane current and its application to conduction and excitation in nerve. J Physiol 117, 500-544 (1952).

66. Huguenard, J. R., Hamill, O. P. \& Prince, D. A.

Developmental changes in $\mathrm{Na}+$ conductances in rat neocortical neurons: appearance of a slowly inactivating component. Journal of Neurophysiology 59, 778-795 (1988).

67. Hamill, O. P., Huguenard, J. R. \& Prince, D. A. Patch-Clamp Studies of Voltage-Gated Currents in Identified Neurons of the Rat Cerebral Cortex. Cerebral Cortex 1, 48-61 (1991). 


\section{SUPPLEMENTARY MATERIALS}

\section{Motor Learning Drives Dynamic Patterns of Intermittent Myelination on Behaviorally-Activated Axons}

Clara M. Bacmeister ${ }^{1}$, Rongchen Huang ${ }^{2,3}$, Michael A. Thornton ${ }^{1}$, Lauren Conant ${ }^{1}$, Anthony Chavez ${ }^{1}$, Alon Poleg-Polsky ${ }^{3}$, Ethan G.

Hughes $^{1,4}$

${ }^{1}$ Department of Cell and Developmental Biology, ${ }^{2}$ Department of Neurosurgery, ${ }^{3}$ Department of Physiology and Biophysics,

University of Colorado School of Medicine, Aurora, CO, USA. ${ }^{4}$ Corresponding author. Email: 1 
bioRxiv preprint doi: https://doi.org/10.1101/2021.10.13.464319; this version posted October 15, 2021. The copyright holder for this preprint (which was not certified by peer review) is the author/funder. All rights reserved. No reuse allowed without permission.

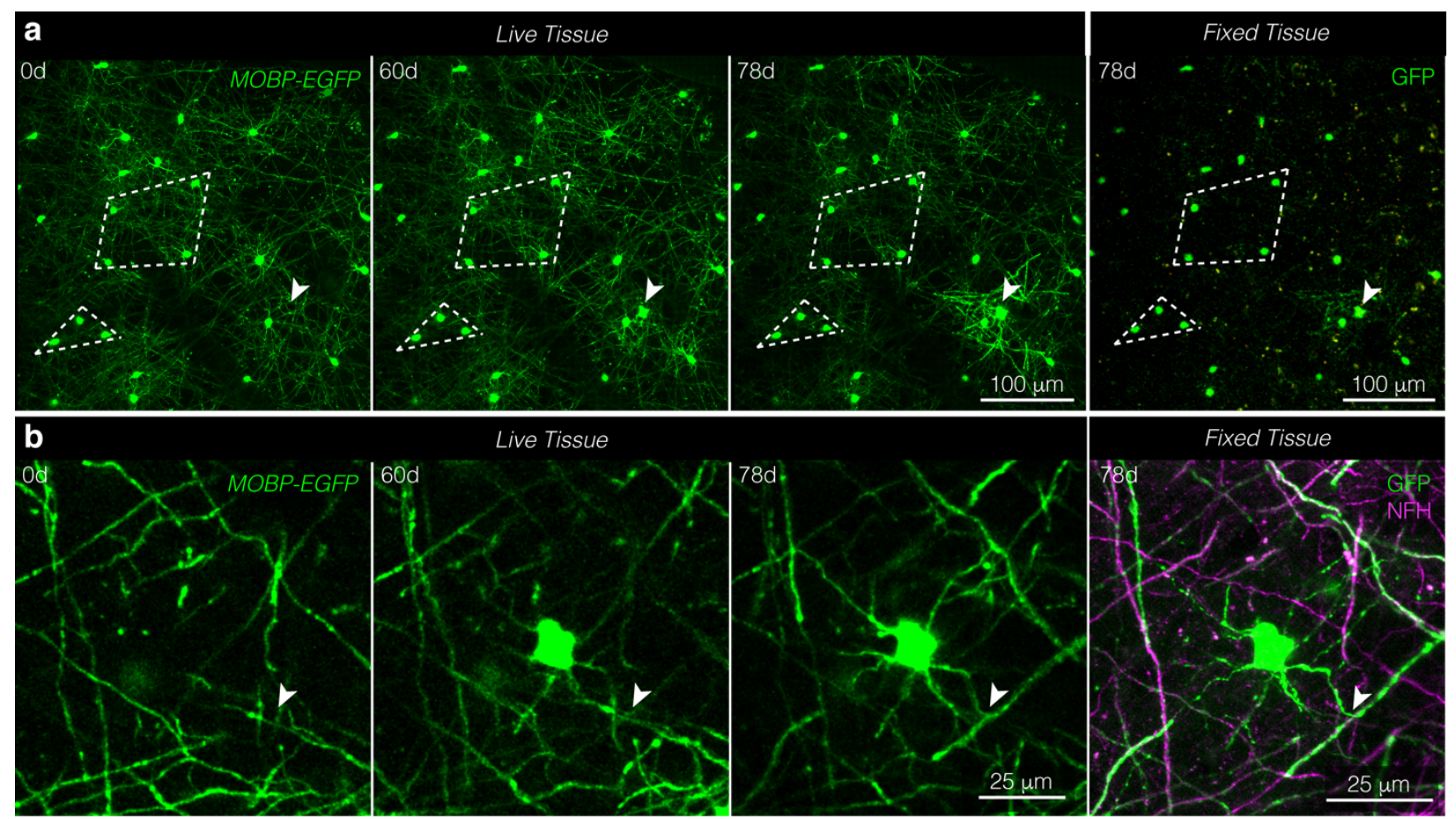

Supplemental Figure 1 Near-infrared branding to identify the same cells in longitudinally-imaged and post-hoc stained tissue. a, The same field of view in live imaged ("Live Tissue", left) and fixed, sectioned, and stained tissue ("Fixed Tissue", right). Patterns of cell bodies (examples outlined in white dotted lines) were maintained across live and processed tissue. Note the new oligodendrocyte generated at $60 \mathrm{~d}$ and delineated with a white arrow. b, A newly generated oligodendrocyte in live ("Live Tissue", left) and fixed, sectioned tissue ("Fixed Tissue", right) stained for oligodendrocytes and myelin (GFP, green) and axons (NFH, magenta). Note the same T-junction marked with the white arrow across live and fixed samples. 
bioRxiv preprint doi: https://doi.org/10.1101/2021.10.13.464319; this version posted October 15, 2021. The copyright holder for this preprint (which was not certified by peer review) is the author/funder. All rights reserved. No reuse allowed without permission.

a

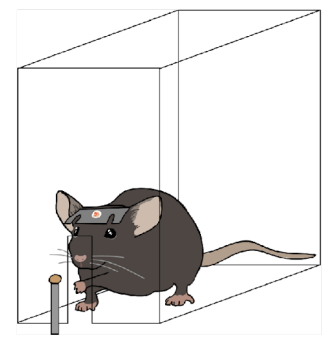

b

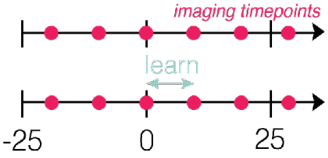

Days post-learning
C

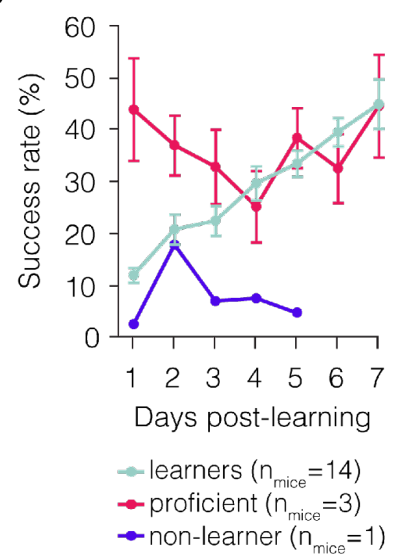

d

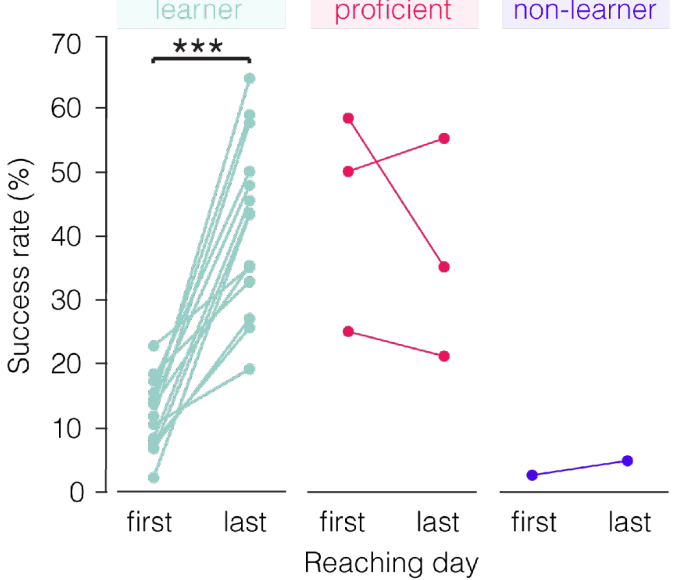

Supplemental Figure 2 Learning trajectory of mice engaging in forelimb reach training. a, Illustration of forelimb reach training box environment. Mice learn to reach by extending their left hand through a slit in a plexiglass box to grab a pellet and return it to their mouth. b, Imaging and training timelines for untrained (top) and learning (bottom) mice. c, The majority of mice learn to perform the forelimb reach task (learners, mint). Learners improve their success rate gradually over the course of seven days of training. In contrast, proficient mice engage in a high percentage of successful reaches and do not improve over the course of training, while nonlearners maintain a low success rate across time and eventually stop reaching altogether. 
bioRxiv preprint doi: https://doi.org/10.1101/2021.10.13.464319; this version posted October 15, 2021. The copyright holder for this preprint (which was not certified by peer review) is the author/funder. All rights reserved. No reuse allowed without permission.

a

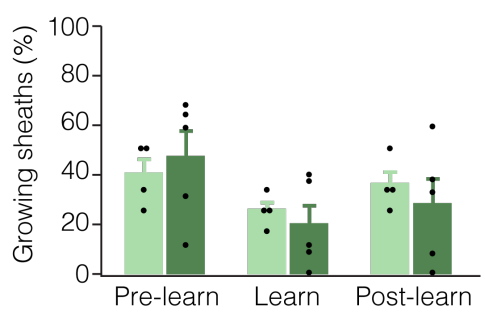

untrained $\left(\mathrm{n}_{\text {mice }}=4\right)$ b

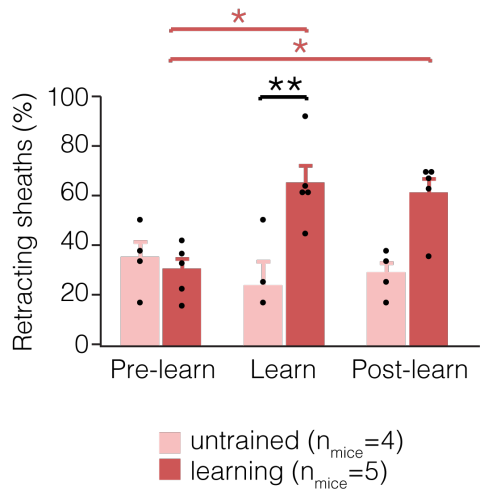

Supplemental Figure 3 Sheath retraction, but not sheath growth, is affected by learning a new skill. a, Proportion of dynamic sheaths engaging in growth before, during and after learning. $\mathbf{b}$, Proportion of dynamic sheaths engaging in retraction before, during and after learning. Learning modulates sheath retraction $\left(F_{3,115.8}=6.76, p=0.0088\right)$. During learning, more sheaths retract relative to untrained mice ( $p=0.0095$; Tukey's HSD) and relative to pre-learning values in trained mice $(p=0.016$; Tukey's HSD). Two weeks after learning, more sheaths retract relative to pre-learning values in trained mice $(p=0.0358$; Tukey's HSD). For detailed statistics, see Supplementary Table 3, Supplementary Figure 3. 

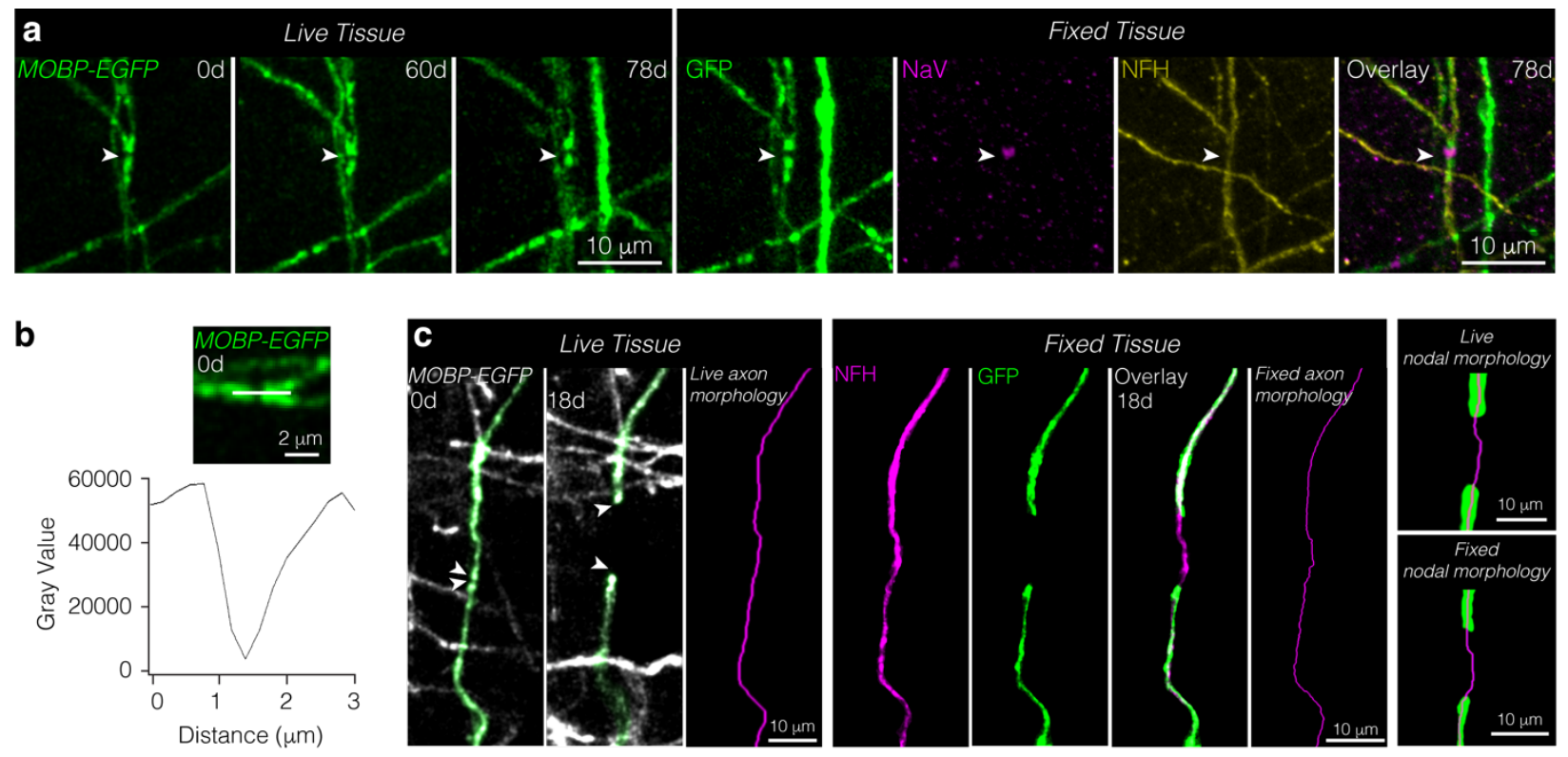

d
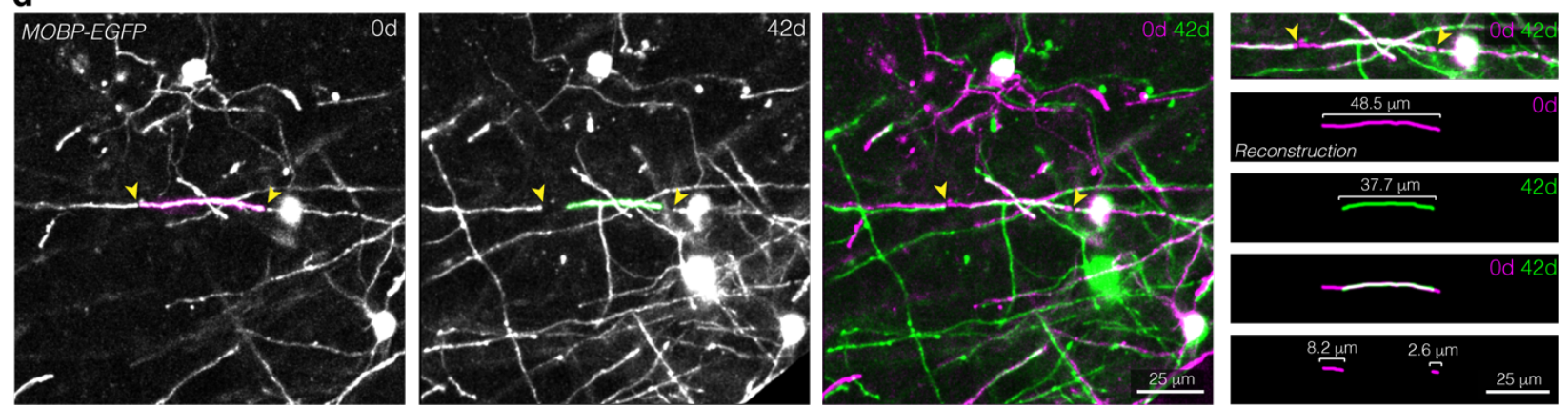

Supplemental Figure 4 ldentifying and tracing nodes and axons across live and fixed tissue. a, The same node in live imaged ("Live Tissue", left) and fixed, sectioned tissue ("Fixed Tissue", right) stained for oligodendrocytes and myelin (GFP, green), sodium channels (NaV, magenta), and axons (NFH, yellow). White arrow points to node across time and in fixed tissue. $\mathbf{b}$, The same node confirmed with $\mathrm{NaV}$ staining in (a) is identified in live imaging by a marked decrease in autofluorescence between two GFP-labeled myelin paranodes. c, Axon morphology is accurately identified in live imaged tissue, as confirmed by post-hoc immunostaining of the same tissue. A lengthening node in live ("Live Tissue", left) and fixed, sectioned tissue ("Fixed Tissue", middle). Axon trajectory is identified using the shape of the myelin sheath prior to node lengthening ("Live axon morphology", magenta) and confirmed using post-hoc immunostaining of the same sheaths for axons (NFH, magenta) and myelin (GFP, green) and reconstructions of the axons ("Fixed axon morphology", magenta). Live node morphology ("Live nodal morphology", top right) is reconstructed and confirmed using posthoc immunostaining and morphological reconstruction ("Fixed nodal morphology", bottom right). d, Sheath length and change in sheath length is determined by aligning fileds of view using markers which extend across the entire duration of the study, including pre-existing oligodendrocytes, which maintain their position throughout the course of imaging (left). Change in sheath length is mirrored by a change in paranode position (right). To determine the change in length, the distance between the initial and final paranodal position is traced using Simple Neurite Tracer and overlayed stacks. 
bioRxiv preprint doi: https://doi.org/10.1101/2021.10.13.464319; this version posted October 15, 2021. The copyright holder for this preprint (which was not certified by peer review) is the author/funder. All rights reserved. No reuse allowed without permission.

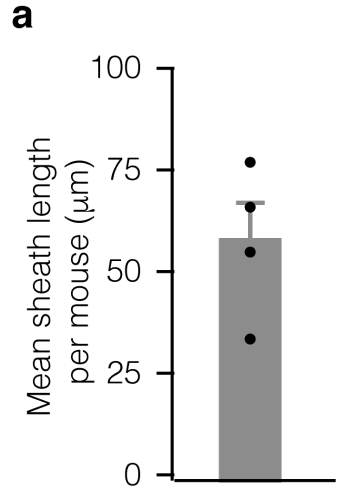

d

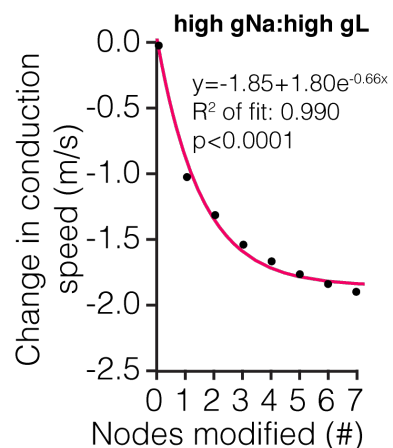

h

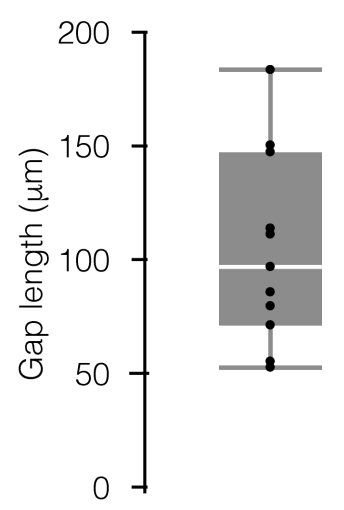

b

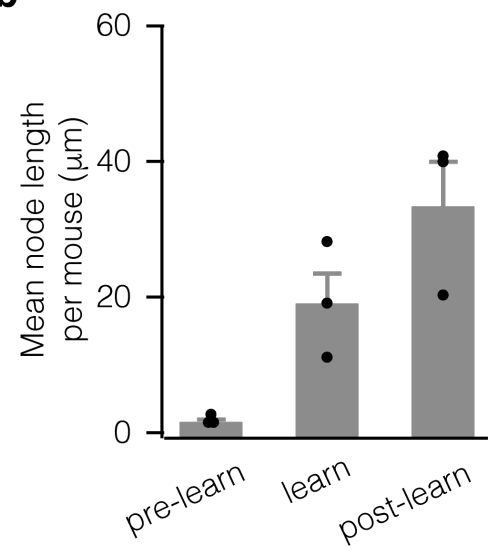

e

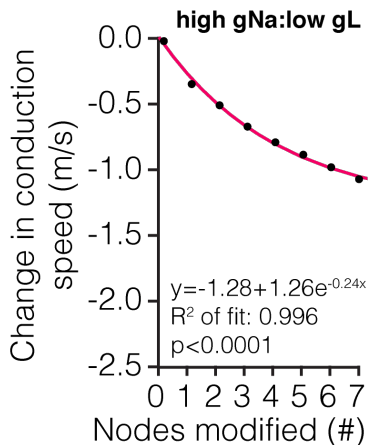

i

Sheaths needed to fill in pre-existing gap

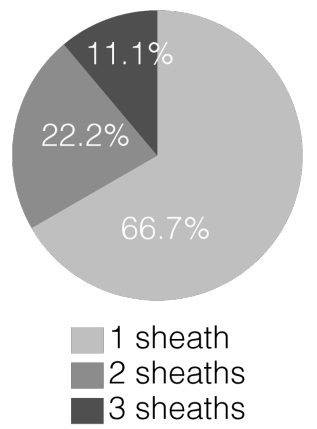

C

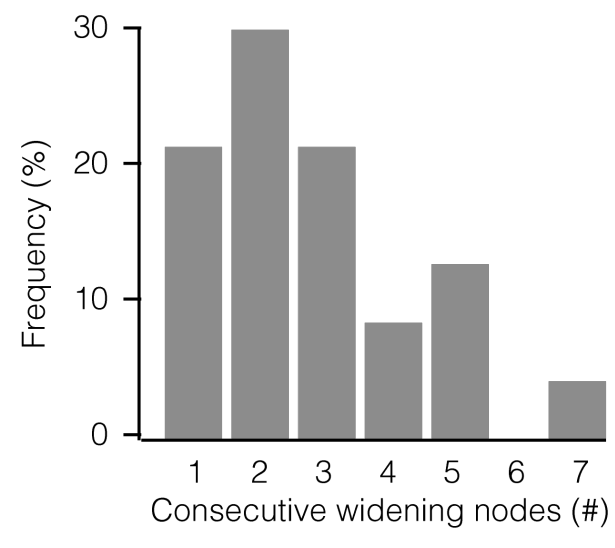

g

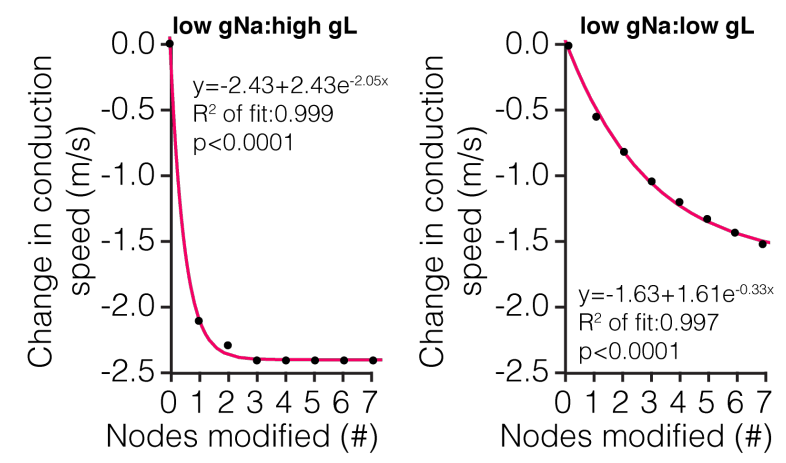

j

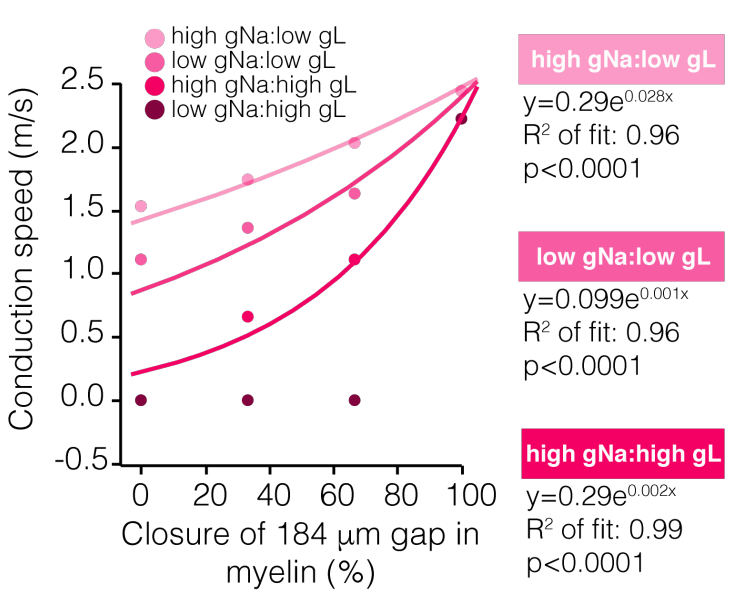

Supplemental Figure 5 Biological correlates of computational modeling data. a, Average pre-existing myelin sheath length per mouse. b, Mean node length of lengthening nodes per mouse, measured across time in pre-learning, learning, and post-learning stages. c, Distribution of the number of consecutive lengthening nodes along a single axon. $\mathbf{d}-\mathbf{g}$, Modeled change in conduction speed as a function of the number of consecutively remodeled nodes at different sodium:leak conductance ratios (node with $=35$ microns, pink exponential fit). Conductance ratios were generated using either $0.4 \mathrm{~S} / \mathrm{cm}^{2}$ (low) or $3.4 \mathrm{~S} / \mathrm{cm}^{2}$ (high) for sodium conductance $(\mathrm{gNa})$ and either $0.01 \mathrm{~S} / \mathrm{cm}^{2}$ (low) or $0.08 \mathrm{~S} / \mathrm{cm}^{2}$ (high) for leak conductance ( $\mathrm{gL}$ ). $\mathbf{h}$, Length of unmyelinated axon in that received new sheath addition following learning, measured from one sheath to the nearest neighboring sheath. Bars and error bars represent median and I.Q.R. i, Proportion of gaps filled by 1, 2, or 3 sheaths following learning. j, Modeled change in conduction speed as a function of proportion of a 184 micron gap filled in by new myelin (pink exponential fit). Conductance ratios as in $\mathbf{d}-\mathbf{g}$. Bars and error bars represent mean \pm s.e.m unless otherwise noted. 
bioRxiv preprint doi: https://doi.org/10.1101/2021.10.13.464319; this version posted October 15, 2021. The copyright holder for this preprint (which was not certified by peer review) is the author/funder. All rights reserved. No reuse allowed without permission.

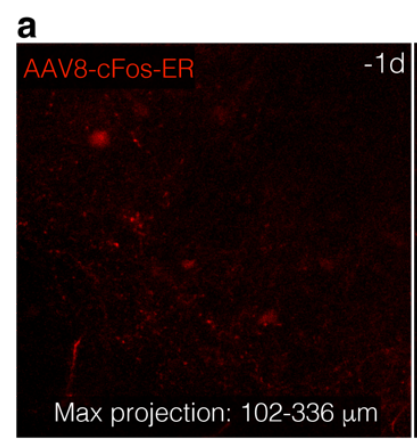

b

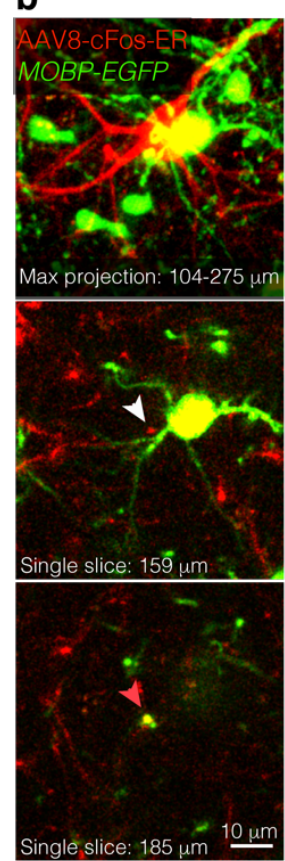

c $3 D$ Projection

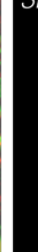
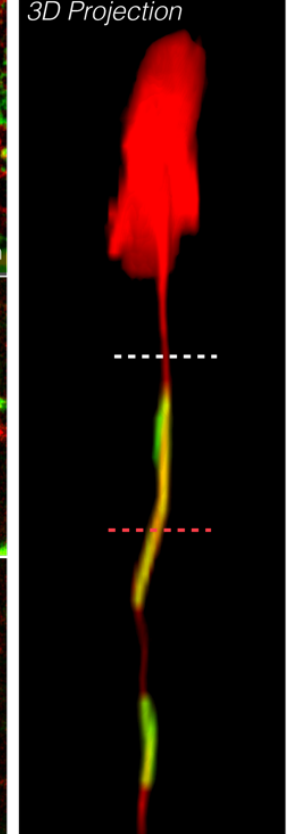

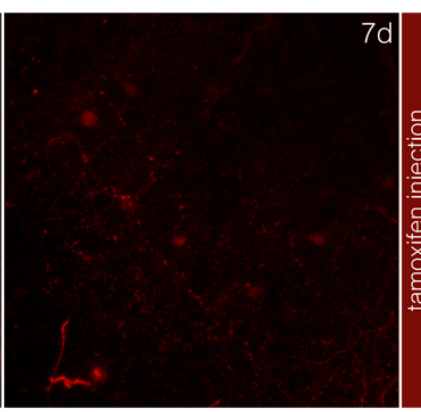

d

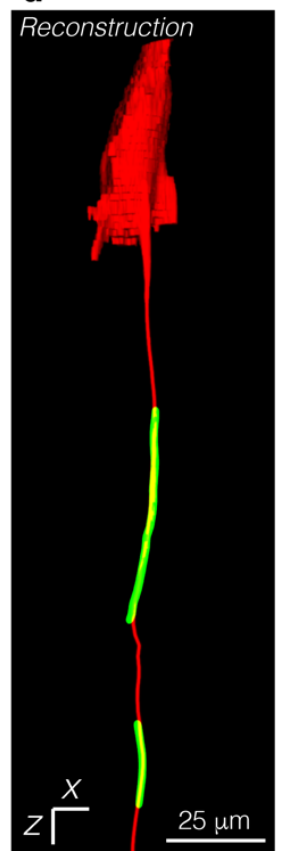

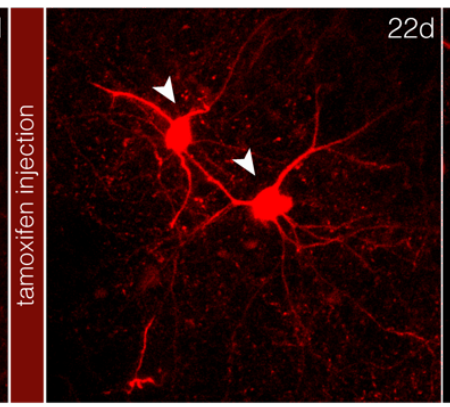

e

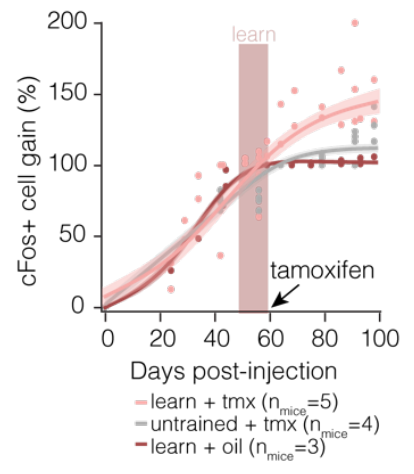

h

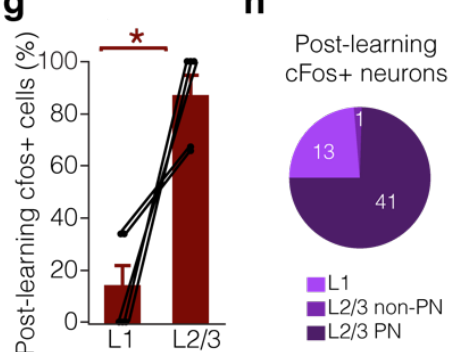

Supplemental Figure 6 Characterizing AAv8-cFos-ER virus. a, Maximum projection of motor cortex injected with the cFos virus 1 day before learning (-1d), one week after learning (7d), three weeks after learning (22d), and 5 weeks after learning (42d). Tamoxifen was injected 3 hours after the final day of learning (7d). b, Maximum projection of a cFos+ neuron (AAv8-cFos-ER, red) and surrounding oligodendrocytes and myelin (in vivo, green) (top). Single slice at 159 microns below the pial surface, with white arrow identifying unmyelinated region of a cFos+ axon (middle). Single slice at 185 microns below the pial surface, with pink arrow identifying a myelinated region of a cFos+ axon (bottom). c, 3D projection of the cFos+ neuron and associated myelin sheaths from (b), with dashed lines corresponding to the single slices in (b). d, The same neuron as in (c), reconstructed using Simple Neurite Tracer. e, Fit curve for modeled change in cFos + cell gain (calculated as \% of pre-tamoxifen cFos+ neuron number) in mice that learn and receive tamoxifen (pink), untrained mice (grey), and mice that learn and are injected with sunflower oil (red). f, Rate of cFos+ cell gain in mice that learn and receive tamoxifen (pink), untrained mice (grey), and mice that learn and are injected with sunflower oil (red). Mice that learn have a heightened rate of cFos + cell gain in the two weeks following learning, while the percentage of cFos + neurons did not change in untrained mice and oil injected mice over the course of the experiment. g, Significantly more cFos + cells appear in L2/3 relative to L1 following learning and injection of tamoxifen. $\mathbf{h}$, The majority of learning-activated cells are putative L2/3 pyramidal neurons (determined by morphology). i, Distribution of traced axon lengths for cFos+ neurons. For detailed statistics, see Supplementary Table 3, Supplementary Figure 6. 